\title{
Risk-based, sensor-fused detection of flooding casualties for emergency response
}

\author{
Kristian Bertheussen Karolius, DNV GL, kristian.bertheussen.karolius@dnvgl.com \\ Jakub Cichowicz, AqualisBraemar ${ }^{l}$, jakub.cichowicz@aqualisbraemar.com \\ Dracos Vassalos, Maritime Safety Research Centre, d.vassalos@strath.ac.uk
}

\begin{abstract}
The intrinsic complexity of the flooding process on ships renders accurate quantification of the flooding risk a highly arduous task, particularly in the context of emergency management, where convolution stems from a multitude of variables, their dependencies and interactions. This is especially true for large cruise vessels, with ever-growing number of passengers, innovative designs and complex internal subdivision. This augments the uncertainty and imposes further challenges on the crew in obtaining a complete overview and making fully-informed decisions following a given flooding event. This paper will present a methodology whereby sensors and analytics are combined utilising probabilistic multi-sensor data fusion to predict the flooding extent with reduced uncertainty to facilitate informed decision-making in emergencies, forming the basis for optimised implementation of emergency response measures for vessel survival and subsequent safe return to port. The framework will be tested with the use of the sensor array onboard an existing large cruise vessel within realistic flooding scenarios. The results demonstrate that the predictions are rapidly converging to the region of the actual damage extent in the presented test-cases, enabling fast and targeted deployment of available mitigation measures with the help of probabilistic supportive evidence. The accurate prediction of flooding extent as presented, is a fundamental prerequisite for, and could be of great assistance in, decision making in emergencies, thus saving lives.
\end{abstract}

Keywords: Damage stability, Flooding emergency response, Life-cycle flooding risk management, Multisensor data fusion.

\section{BACKGROUND AND OVERVIEW}

\subsection{An industry in change}

The cruise segment has seen a relentless upsurge in the last decades, where new megaships continue to outsize older generation of designs. This trend has been driven by economy of scale, offering a competitive edge to ship-owners and operators in an ever-burgeoning market. The new giants of the sea and their ever-growing number of passengers, unparalleled proportions, design innovations and complexity are subsequently increasing the associated risks and posing challenges to the vessel operators in terms of risk management. Seen in light of recent accidents (MIT, 2013), human cognition and lack of situational awareness is identified as a serious weakness. Recognition of the evolving risks has led to an intensive pursuit for more optimal design solutions and has facilitated the introduction of riskbased ship design methodologies and subsequent cost-effective risk control options aiming at both prevention and post-accident mitigation and control (Papanikolaou et al., 2009). The latter is directly related to emergency response but is, however, largely dependent on human intervention to perform their intended function, focusing mainly on controlling and containing situation-specific flooding processes. In the absence of proper situational assessment the process being controlled is rather hypothetical and may be significantly different from the actual one. This may result in the implementation of suboptimal or even the wrong mitigation measures. In the case of the latter the action intended to improve the situation may in fact worsen it, resulting in severe consequences. Increased surveillance and monitoring, (e.g. the introduction of flooding sensors in passenger vessels as required by SOLAS (2009) Reg. II-1/22-1), aims at reducing the uncertainty in order to assist the crew in correct and timely decision-making. However, their potential in providing accurate information, particularly in emergencies, has yet to be fully taken advantage of, using more advanced analytical approaches.

\subsection{Life-cycle risk management}

Risk management is a life-cycle process, entailing risk reduction, mitigation and control by design, managing residual risks in normal operations and crises management in emergency situations (i.e. emergency response). A generic framework for

\footnotetext{
${ }^{1}$ Formerly affiliated with the Maritime Safety Research Centre
} 
addressing life-cycle risk systematically is lacking in the maritime industry, stemming from a history of being largely rule based, following minimum (often deterministic) standards of safety, thus failing to nurture a more comprehensive risk management framework. For life-cycle flooding risk management, the accident prevention, or post-accident mitigation and control safety measures must be monitored and assessed during the vessel life-cycle, thus ensuring tolerable risk levels. More specifically, in the case of a flooding emergency, the various safety barriers are available and working optimally as intended. More comprehensive monitoring would further provide extensive data for feedback and continuous improvement through both passive (design) and active means (operation).

The inherent complexity of the flooding process and the lack of a comprehensive life-cycle (flooding) risk management framework for large passenger vessels has in recent years been recognised. The probabilistic damage stability regulations in Reg. II1/6-8 of SOLAS 2009 (IMO, 2006) aim at assessing a vessels design envelope and provide a safety baseline (A-index), however, are largely simplified for a unified and easy application throughout our industry. Attempts have also been made to utilise emerging technology to improve the real-time situational awareness in operation and emergencies. Two notable examples are the operational flooding vulnerability measure suggested by Jasionowski $(2010,2011)$ and a method for breach detection from floodwater inflow-rate as suggested by Napa Ltd (Penttilä et al., 2010, Ruponen et al. 2007, 2012, 2015 $\&$ 2017). Both these novel contributions are highly valuable, however, they are either too generic in providing an overall vulnerability that covers all possible cases rather than the actual case being realised (limited to the operational phase, e.g. Jasionowski), or completely deterministic not catering for any of the natural variability of such a stochastic problem (limited to emergency phase, e.g. Napa Ltd). In this respect, a more exhaustive and fully probabilistic solution for Life-Cycle Risk management would be beneficial, bridging the gap between all the life-cycles, namely design, operation and emergencies.

\subsection{Research focus}

The research presented in this paper emanates from Ph.D. research (Karolius, 2019), directed at the development of a framework where sensors and analytics serve to improve quality of information in risk assessment and, hence, to provide means for a more comprehensive life-cycle flooding risk management framework for large cruise vessels. This paper outlines the developed framework with focus on application in the emergency phase, which address the problem of determining with high confidence the extent and location of damage following hull breach in a collision incident. Utilising probabilistic supportive evidence for quantified decision making, the framework would provide improved survival assessment and enable the crew to implement emergency response in a timely, targeted and efficient manner. This should prolong the time available for safe and ordered evacuation, potentially facilitate safe return to port.

The methodology, based on Bayesian inference, will use available statistics as a-priori information and sensor data as continuously updated evidence. This entails the analysis of the flooding process and damage stability characteristics to identify the most relevant variables and to develop a-priori distributions and corresponding probabilistic likelihood functions applicable to the multi-sensor framework combining the state-of-the-art timedomain simulation software tool PROTEUS3 (Jasionowski, 2001) and available statistics. The methodology will be tested with the use of sensors onboard an existing large cruise sample vessel (sensor layout as built) within flooding emergency response scenarios.

To enable testing, the methodology has been implemented within a demonstration platform, which reads emulated sensor data and provides sequentially updated assessments of the likely damage location and extent. Time-series from time-domain simulations are interpreted as actual sensor readings; as in a real emergency, the readings are affected by the environment and dynamic responses of the damaged ship. Although application in emergencies are in focus, implementation in the remaining lifecycles will also briefly be discussed. 


\section{UNCERTAINTY}

The main objective of the developed methodology is to reduce uncertainty of prediction in flooding emergencies. Traditional approaches recognise two main categories of uncertainty, namely aleatoric, and epistemic uncertainty (Schweder \& Hjort, 2016). The terms aleatoric variability and epistemic uncertainty, or simply variability and uncertainty, are also used for the respective categories (Bitner-Gregersen et al., 2012). The variability (aleatoric - alea - Latin - the game of dice) is a natural property of the flooding process due to the random character of the excitation (wave loads) and the vessel response. Variability cannot be reduced by additional data collection because it is a natural property of the variable in question and is always there. The uncertainty (epistemic - Greek - knowledge) represents the level of knowledge about the system being modelled and it can be reduced by further data collection. Typically, we may be uncertain about parameters being used in a model (probability distribution) of the system. Epistemic uncertainty may have a range of sources, and may be classified into the following categories (Skjong et al., 1995):

- Measurement Uncertainty due to imperfection of an instrument used to register a quantity.

- Statistical Uncertainty due to limited information such as a limited number of observations of a quantity.

- Model Uncertainty due to imperfections and idealisations made in physical model formulations as well as in choices of probability distribution types for representation of uncertainties.

The measurement uncertainty is highly related to sensors, sensor reliability and their ability to ensure accurate measurements of the process of interest. A sensor-based measurement accuracy is, in accordance with ISO 5725-1 (ISO, 1994), divided into two categories, namely Trueness and Precision, where "Trueness" refers to the closeness of agreement between the arithmetic mean of a large number of test results and the true or accepted reference value, while "Precision" refers to the closeness of agreement between test results (Figure 2-1).

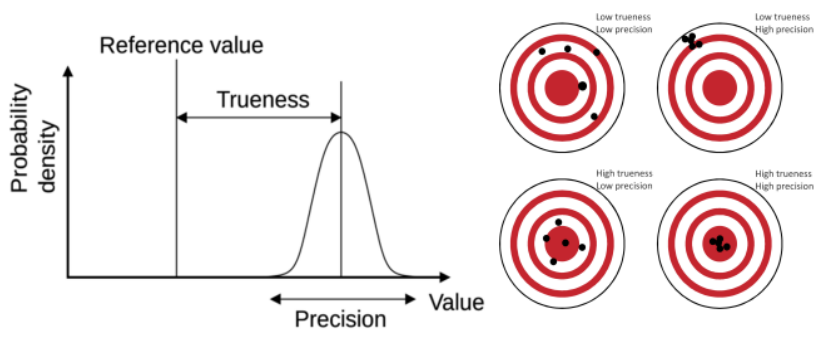

Figure 2-1: Visualisation of trueness and precision (DNV GL, 2018),

The main focus of the research presented herein is on the aleatoric variability inherent in the flooding process in a dynamic environment. This, in effect, justifies the particular effort allocated to techniques allowing the use of Bayesian inference and multisensor data fusion to obtain a strengthened belief on the potential variability of the process. Epistemicuncertainty in the form of sensor measurement uncertainty, false negatives and positives, have also been implemented for selected sensor types.

\section{MULTI-SENSOR DATA FUSION}

"Data fusion is the process of combining information from a number of different sources to provide a robust and complete description of an environment or process of interest"

(Durant-Whyte \& Henderson, 2016).

The process of fusing, or aggregating, scattered information from a range of independent sensors is important in applications where large amount of data must be combined, to obtain information of quality adequate for supporting decision-making. Data fusion is applied in a wide range of industries, such as military systems, surveillance and monitoring systems, process control systems, and information systems. It also plays a central role in autonomous systems and robotics because it allows essential measurements and information to be combined to generate knowledge with high level of confidence (or lack of uncertainty), to enable decisions to be executed autonomously. Due to the complex and multivariate nature of the problem, multi-sensor data fusion process is highly relevant for producing strengthened knowledge for optimised decision making in flooding emergences. 


\subsection{Reasoning behind data fusion}

In all data fusion problems, there exists some process or quantity whose actual state or value cannot be known exactly with the use of a single and direct source of information. Information is, therefore, obtained indirectly from various related sources and the actual state is predicted under uncertainty (inferred). In relation to the problem of a flooding incident, its extent cannot, in the general case, be obtained from a single measurement or direct source and must be deduced from a range of flooding sensors from various compartments. Furthermore, the damage extent is not determined by the breach alone, but it also depends on the status of internal openings and subsequent progressive flooding, hence additional sensor observations may be used in order to increase confidence of the prediction. To enable utilisation of multi-sensor fusion techniques, the problem needs to be defined explicitly. Formally, this can be expressed by means of the following definitions adopted from Durant-Whyte \& Henderson (2016).

- The quantity, or process of interest can be represented by variable, $x$ which may assume any value from the set $X$ of possible states; $x \in X$, where $X=\left[x_{1}, x_{2}, \ldots, x_{n}\right]$. The current state of $x$ can be simply denoted as: $x=x_{i}$.

- Sensor observations are made to gain more knowledge of the state of $x$. Such sensor observations can be represented by $z$, which is contained in the set $Z$ of possible observations, i.e. $z \in Z$, where $Z=\left[z_{1}, z_{2}, \ldots, z_{n}\right]$. An observation made of $z$ corresponds to a single value of the set $Z$, i.e. $z=z_{i}$.

- For an observation $z$ to be of any value, its relation to the quantity or process of interest is needed. That is, for every specific quantity or process, $x=x_{i}$, an observation model exists that describes possible observations in $z \in Z$. More specifically, for each specific $x$ there exist a set of observations $z$.

- Given an observation $z=z_{i}$ from a sensor, the main goal is to infer the actual state of the quantity or process of interest, $x$. For this purpose, a mapping model is needed that maps observations to states of the quantity or process of interest, i.e. $f\left(z=z_{i}\right) \rightarrow x \in X$. This mapping is performed by the likelihood functions (also termed sensor model).

\subsection{Probabilistic sensor fusion}

The probabilistic sensor fusion implemented in this development, utilises Bayesian inference, which entails modifying conditional probabilities. At the heart of Bayesian inference lies Bayes theorem (Bayes, 1763). It uses a-priori probability distributions, which are updated once new evidence emerges to posterior probability distributions with the help of likelihood functions and the laws of probability. The idea of likelihood functions pairs perfectly with a system relying on sensor information. Knowledge is always uncertain to some degree, but we may alter our beliefs based on the strength of the available evidence, which is exactly what Bayes theorem represents. Bayes theorem encodes variables $x$ and $z$ as a joint probability distribution $P(x, z)$ (Figure 3-1). Applying the chain rule of conditional probability with respect to both variables, $\mathrm{x}$ and $\mathrm{z}$, separately, results in Eq. 3-1 and Eq. 3-2. Combining these two expressions and rearranging leads to the well-known Bayes theorem; Eq. 3-3. The power of Bayes theorem lies in its ability to provide the conditional probability of $x$ from known evidence, $z$.

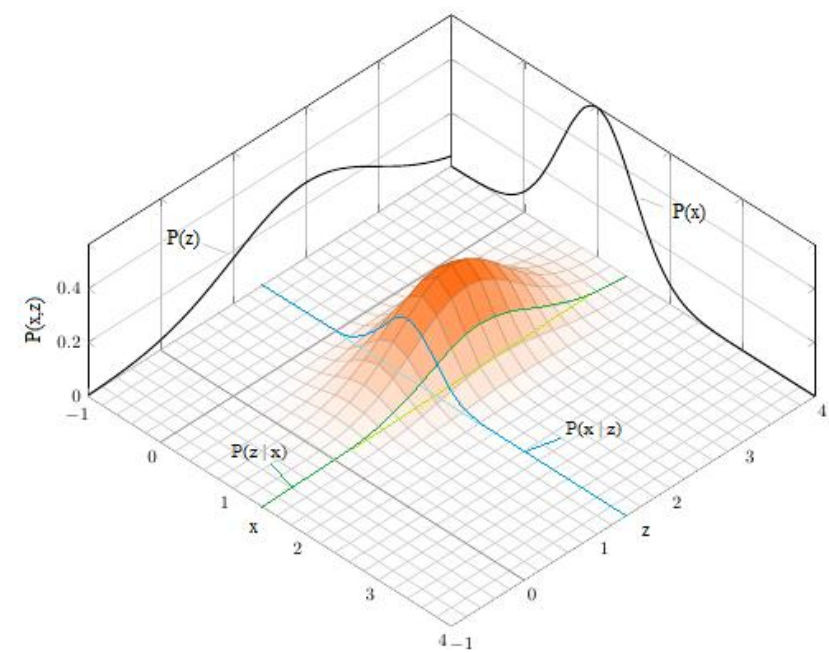

Figure 3-1: Joint, marginal and conditional probability distributions.

$$
\begin{aligned}
& P(x, z)=P(x \mid z) P(z) \\
& \text { Eq. 3-1 } \\
& P(x, z)=P(z \mid x) P(x) \\
& \text { Eq. 3-2 } \\
& P(x \mid z)=\frac{P(z \mid x) P(x)}{P(z)} \\
& \text { Eq. 3-3 }
\end{aligned}
$$


In direct translation to sensor fusion, the belief about a state of a quantity, or process of interest, $x$, may be inferred from sensor observations, $z$. In practical application, however, the likelihood function alone serves the role of a sensor model, or mapping between the observations and the actual state of the process or quantity in question, $x$, whilst the marginal evidence $P(z)$ is only acting as a normalising constant. On this basis, the theorem could be rewritten as in Eq. 3-4, where $C$ represents the normalising constant.

$$
P(x \mid z)=C P(z \mid x) P(x) \quad \text { Eq. 3-4 }
$$

The above clearly illustrates the advantage offered by Bayes theorem in a sensor fusion framework, as it provides a direct means of combining observed information with a-priori belief about the state of the process or quantity of interest. The observed information in this case is fragmented, collected from various sensor arrays, and fused into a new, more complete, belief of the state of $x$. Multiple sensor observations from an array of sensors is represented by Eq. 3-5. The likelihood function for the sensor observations is represented by Eq. 3-6 and by employing Bayes theorem, we obtain Eq. 3-7.

$$
\begin{array}{ll}
Z=\left[z_{1}, z_{2}, \ldots, z_{n}\right] & \text { Eq. 3-5 } \\
P(Z \mid x)=P\left(z_{1}, z_{2}, \ldots, z_{n} \mid x\right) & \text { Eq. 3-6 } \\
P(x \mid Z)=C P\left(z_{1}, z_{2}, \ldots, z_{n} \mid x\right) P(x) & \text { Eq. 3-7 }
\end{array}
$$

In practice, it would be difficult to do this directly because it would require the knowledge of the joint distribution $P\left(z_{1}, z_{2}, \ldots, z_{n} \mid x\right)$. This in turn, entails knowing the joint distribution of all possible combinations of observations conditioned on the underlying state. To resolve this, it is possible to take advantage of the concept of conditional independence, which plays a significant role in constructing the fusion algorithms for the framework. In order to illustrate this, we may consider a state of a process or quantity $x$. If two observations were made of the state of $x$, namely $z_{1}$ and $z_{2}$, it is obvious that the two observations are not independent as they both depend on the common state of $x$. This is represented by Eq. 3-8.

$$
P\left(z_{1}, z_{2}\right) \neq P\left(z_{1}\right) P\left(z_{2}\right) \quad \text { Eq. 3-8 }
$$

However, it is reasonable to assume that the observations are independent if the true state of $x$ is known, i.e. the observations $z_{1}$ and $z_{2}$ are conditionally independent given the state of $x$, which is represented by Eq. 3-9.

$$
P\left(z_{1}, z_{2} \mid x\right)=P\left(z_{1} \mid x\right) P\left(z_{2} \mid x\right) \quad \text { Eq. 3-9 }
$$

This would not be a bad representation of the true state of $x$; simply what the two observations $z_{1}$ and $z_{2}$ have in common. Assuming conditional independence allows, in the case of two sensor readings $z_{1}$ and $z_{2}$, using Eq. 3-10. This could be further generalised for a range or array of $n$ sensors, as in Eq. 3-11.

$$
\begin{array}{ll}
P\left(x \mid z_{1}, z_{2}\right)=C P\left(z_{1} \mid x\right) P\left(z_{2} \mid x\right) P(x) & \text { Eq. 3-10 } \\
P(x \mid Z)=C \prod_{i=1}^{n} P\left(z_{i} \mid x\right) P(x) & \text { Eq. 3-11 }
\end{array}
$$

\subsection{Sequential updating}

Eq. 3-11 represents a single posterior update from two sensor sources. Data obtained from actual sensor readings arrives continuously in real-time. From this data stream, we want to update the posterior belief of our unknown state of interest, $x$, in real-time as new information is made available at each time step $k$. Direct application of Eq. 3-11 would require storing of all past sensor data $z^{k-1}$, and implementing this in the posterior update. However, we may express Eq. 3-11 in recursive form and take advantage of the fact that previous data is already present in the posterior distribution from the past time-step $k-1$. The posterior update in time can be interpreted as a recursive state transition of a Markovian process (Gagniuc, 2017) in which the next state $x_{k}$ depends solely on the immediately preceding state $x_{k-1}$. This results in the recursive form of Bayes formula represented by Eq. 3-12

$$
P\left(x \mid Z^{k}\right)=C \prod_{i=1}^{n} P\left(z_{k_{i}} \mid x\right) P\left(x \mid Z^{k-1}\right) \quad \text { Eq. 3-12 }
$$

In this recursive representation, we notice that at time $k$ we only need to keep a representation of the current state and may otherwise ignore the past. The advantage is therefore that we only need to compute and store the posterior likelihood $P\left(x \mid Z^{k-1}\right)$ which contains a complete representation of the past information. When new information is made 
available in the form of $P\left(z_{k} \mid x\right)$, the previous posterior takes the role of the current prior and the normalised product of the two becomes the new posterior $P\left(x \mid Z^{k}\right)$. Therefore, Eq. 3-12 represents a significant improvement in computational and memory requirements over Eq. 3-11.

\section{PROBABILISTIC MODELS}

Sensor fusion relies on probabilistic models in the form of a-priori distributions, representing the apriori belief (i.e. without any supportive evidence) of the process or quantity in question and sensor models (likelihood functions) representing a mapping between the variable and available evidence (sensor readings). The latter simply determines the likelihood of obtaining a specific sensor reading, $z$, conditioned on the fact that the state of $x$ has assumed a particular value. The following section discusses the relevant variables governing the flooding process and sensors available for use in the developed framework. This is followed by a more detailed discussion on the development of the respective probabilistic models.

\subsection{Variables and sensors}

Variables governing the flooding process may be roughly divided between breach-, vessel- and environmental-variables. The breach variables comprise the geometrical properties of the damage opening that connects internal spaces to the sea, resulting from the collision (or grounding/contact). They comprise (Figure 4-1) size variables, namely: length, height and penetration depth (designated by $L$, $H$ and $Y$ ) and position variables: longitudinal and vertical (designated by $X$ and $Z$ ).

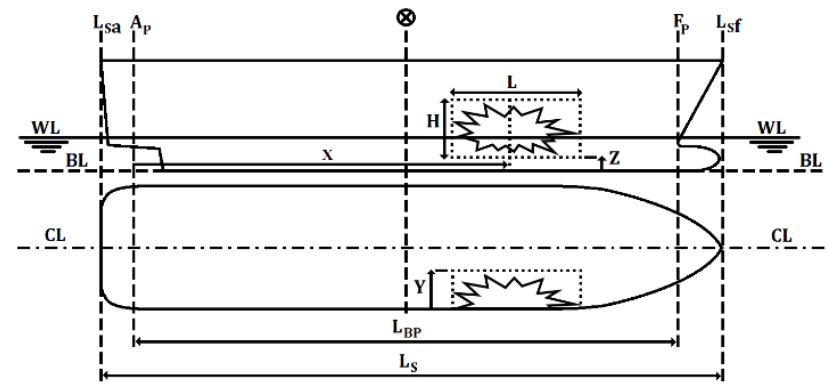

Figure 4-1: Damage breach geometrical properties.

Obviously, a real breach is unlikely to be completely regular in shape due to the structural dynamics and mechanics involved in the collision but are necessary simplifications to enable the development of statistics describing the breach geometry as initially introduced by Lützen (2001, 2002) and further discussed in section 4.2.1 of this paper. The vessel variables comprise a set of parameters from both vessels involved in a collision scenario, namely the struck and striking vessels (both may be subjected to a breached hull and subsequent flooding). The internal subdivision will determine how the breach opening will translate into the initial damage extent (i.e. the initial loss of buoyancy). The internal arrangement comprising internal openings such as ducting, piping, doors, hatches, windows, shafts, etc., connecting compartments will further influence the flooding progression. This compartment connectivity is dynamic, as openings such as doors and hatches may be opened and closed during the operation of the vessel for specific periods of time or leak or collapse under the floodwater pressure. The possible progression of floodwater through the internal openings gives rise to the progressive damage extent (Figure 4-2).

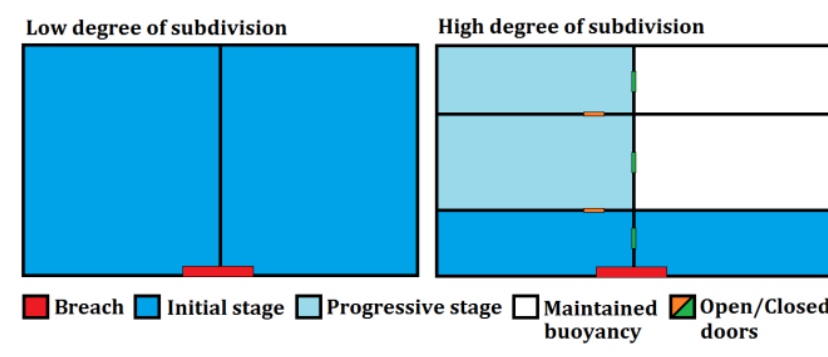

Figure 4-2: Initial and progressive stage of damage dependent on degree of internal subdivision and internal opening status.

The progressive extent is often divided onto several subsequent stages depending on the sequence of openings being submerged and adjacent compartments progressively flooded. Supportive evidence of the complete damage extent may be obtained from available flooding sensors and door status sensors as required by SOLAS Reg. II-1/22-1 (IMO, 2006) and SOLAS Reg. II-1/13-6 (IMO, 2006) respectively, and is implemented in the fusion framework to support inference on the flooding extent. The interplay between the striking- and struck vessels will influence the damage breach according to the direction and magnitude of the impact force (energy transfer). Relevant variables involve vessel's speed, mass and heading as well as structural 
crashworthiness and specific geometric features, in particular the striking vessel's bow shape and height. Modelling of vessel variable dependency in collision damages and crashworthiness was addressed by a number of publications, such as Pedersen (1999 \& 2010), Lutzen (2001), Törnqvist (2003) and Paik \& Sea (2007). Vessel geometry and structural crashworthiness are design variables, hence they are set (invariant) and outside of the operator's control in an emergency. However, the details of the striking ship involved in a collision scenario, such as displacement, speed, heading, and bow height would provide a useful indication in the assessment of the breach extent. In most of the cases such information can be retrieved (at least partly) from the Automatic Identification System (AIS) required by SOLAS Reg. V/19 (IMO, 2009) and is incorporated as supportive evidence into the fusion framework to support inference on the breach extent.

A range of parameters associated with the vessel's loading condition (lightship weight, cargo and tank content) will determine its centre of gravity ( $\mathrm{CoG})$, which in combination with the hull-form will govern the floating position, righting moment and dynamic responses in waves. The floating position will affect the vertical position of the breach opening in relation to the baseline following a collision. Shallow-draught stricken ships (e.g. cruise ships) are expected to suffer collision damages extending to much lower compartments than the deep-draught ships (e.g. laden tankers) although cargo ships may not have as many decks below the bulkhead deck. Draught of the vessel would provide additional supportive information in assessing the vertical damage extent. A vessel's loading condition is known by the operators from the onboard loading computer, as required by SOLAS Reg. II-1/8-1.3 (IMO, 2009). Furthermore, modern ships, such as the sample vessel utilised in this paper, have pneumatic draft sensors, which will provide a continuous reading of the vessel draught, heel and trim. This information is incorporated into the fusion framework to support inference on the vertical damage extent. Trim has been disregarded on the premise that large cruise vessels operate within a very narrow trim range but it could be readily included within the development if necessary.
Regarding the environmental variables, the focus is mainly on waves as, particularly in high sea states, they produce non-linear dynamic responses of the vessel. In damaged condition, the waves cause complex, coupled dynamic interactions between the sea, floodwater and the damaged ship, strongly affecting vessel survivability. Furthermore, although the floodwater will in many cases result in subdued vessel motions, due to its added damping and added inertia effects (as in the case of anti-rolling tanks), the flooding has always detrimental impact of the vessel's survivability. The passing waves will also affect the vessel stability by dynamic changes to underwater volume (and waterplane area) and subsequent variations of the righting arm, GZ. Even more significant is the "pumping" action of waves causing floodwater accumulation in damaged spaces and on low-freeboard decks that would not be flooded in calm water. This results in multiple free-surface effects and progressive flooding through nonwatertight openings, further adding to the complexity of the ship and floodwater responses.

Knowledge of the wave height provides supportive evidence on the possible progressive flooding routes and is implemented within the framework. For this purpose, it is assumed that significant wave height at the area of operation is known either from the wave radar (Grønlie, 2004) or data from wave -buoy or -satellite imaging for the specific location. Although wind forces create considerable heeling moments, they have traditionally not been systematically accounted for when considering a damaged ship, being colinear in most situations. Hence, in real beam-seas (considered to be most severe in waves) the wind force would help resist capsize rather than strengthen the action of waves (Papanikolaou, 2007). Wind sensors (anemometers) are today fitted on all operating vessels, in line with SOLAS Reg. V/5 (IMO, 2006) and provide speed and direction of the wind acting on the vessel. However, by implementing evidence from the pneumatic draft sensors, the dynamic floating position (heel and trim), would indirectly reveale the effect the induced wind-heeling-moment impose on the vessel through the vessel floating position. 


\subsection{A-priori distributions}

\subsubsection{Initial damage extent}

Damage extent is a combination of the initial and progressive damage extents. The breach geometry directly defines the initial damage extent and available damage statistics may be utilised to fit appropriate probability distributions. Subsequently Monte Carlo (MC) sampling of pertinent distributions of damage statistics can be used to identify initial damage extents. The development of the probability distributions for geometrical properties of a breach utilises the database of ship casualty data from project GOALDS (Bulian, 2011), which is an update of the HARDER database (Lutzen, 2002). The latter project is a basis for the probabilistic damage stability framework of SOLAS. However, as discussed in the following, there are some limitations in utilising the SOLAS distributions as a-priori distributions within a sensor fusion framework. Firstly, the distributions were simplified to enable harmonisation for all ship types and may therefore lack important ship-type dependent properties. This involves covering the conditional dependencies of certain variables, e.g. vessel draft. Further simplifications are imposed to facilitate the use of the data for the regulatory framework.

For example, the SOLAS distribution for the longitudinal damage position has been linearised and is, therefore, disregarding the larger concentration of damages in the forward region. It is further assumed that the breaches always have the lower vertical limit below the waterplane, as to ensure flooding (being a static consideration conditioned on water ingress). Where possible the distributions developed for the purpose of the framework are free from such assumptions, e.g., the breach may fully be located above the waterplane and still cause flooding due to the action of waves. Moreover, although at this stage the development of a-priori distributions is based on the dataset for collision damages only, the methodology could be easily adopted and implemented for grounding damages as well. Furthermore, the variables have not been nondimensionalised to prevent overestimation of the variables when scaled up to large cruise vessels (approximately $70 \%$ of the data comes from vessels below $150 \mathrm{~m}$ in length). The probabilistic models for the initial damage extent are derived in the statistical software R (R Core team, 2013), supported by a range of libraries. Copula-theory have been used for the joint distributions, with the optimal copula identified from a range of copula-families. For details of the developed distributions, see section 6.2.1 of Karolius (2019). In summary, the relevant dependencies are identified and applied as in the following:

- The longitudinal damage position, $X$, has been scaled to correspond to a given ship subdivision length $\left(L_{S}\right)$, as only breaches manifested on the vessel hull is of interest, i.e. $X \in\left[L_{S_{a f t}}, L_{S_{f w d}}\right]$.

- To ensure that breaches are manifested on the vessel hull only, a rejection sampling should be performed when sampling the damage length, $L$ using the provided boundary function.

- The expected vertical damage position, $Z$, is found to be dependent on the vessel draft, and therefore linearly parameterised accordingly.

- The available data indicate that the damage height, $H$, is conditional on the vertical position of the damage, $Z$, (Figure 4-3) and that the damage penetration, $Y$, is dependent on the damage length, $L$ (Figure 4-4).

Having a complete set of distributions, MC sampling may be used to draw random breaches and identify what compartments are compromised. Sufficiently large sample ensures that all possible initial damage extents (i.e. collections of rooms) are accounted for and the frequencies of occurrence of the individual extents within the sample can be used to estimate their probabilities. For simplicity, the process is demonstrated for the starboard side breaches. A large sample of 1,000,000 breaches was generated in $R$ to ensure convergence and coverage of all possible initial damage extents. The sampled breaches were checked for overlap in geometrical properties against every compartment in the vessel arrangement. The 1,000,000 sampled starboard breaches resulted in 19,225 unique initial damage extents, which together with corresponding probabilities represent the a-priori belief of possible initial damage extents. 


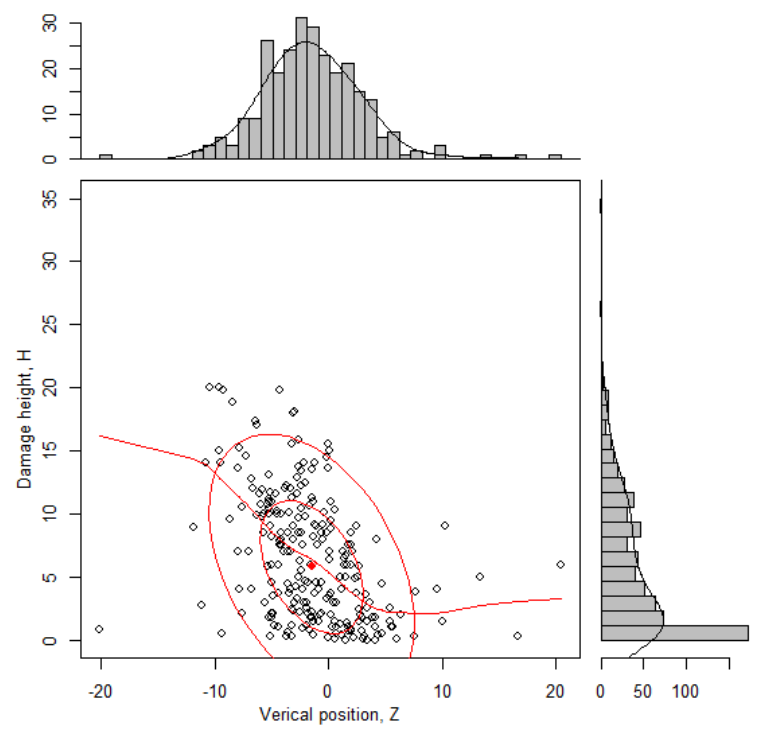

Figure 4-3: Joint dependency between $\mathrm{Z}$ and $H$.

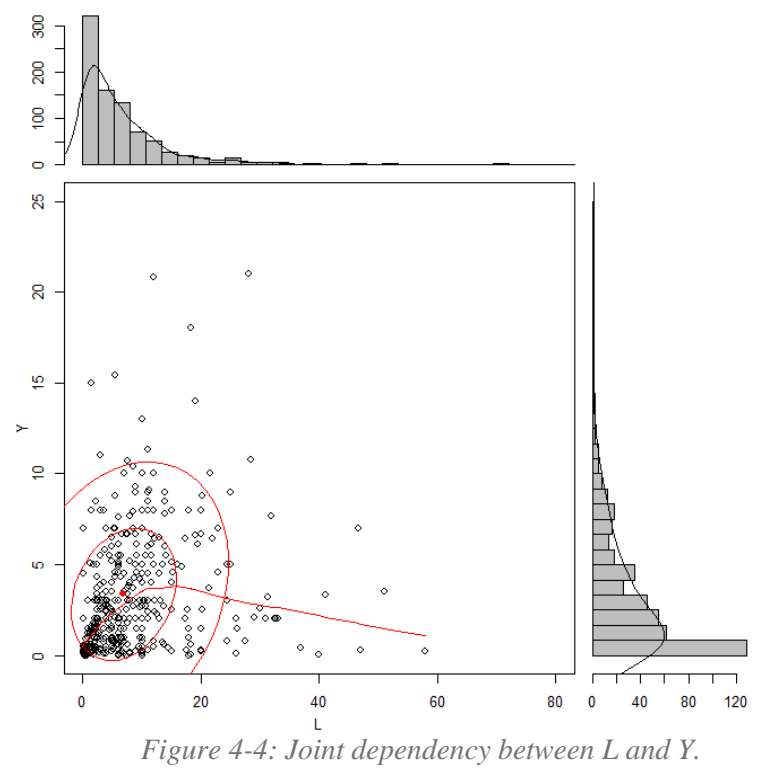

\subsubsection{Opening frequencies}

Statistics for door open/closed frequencies are available for main watertight doors from status sensors required by SOLAS Reg. II-1/13 (IMO, 2006). Non-watertight doors are normally not fitted with such sensors. In absence of status sensors the opening frequencies for non-watertight doors may be estimated from crew/passenger traffic data. Door opening status may be modelled by a Bernoulli process (Eq. 4-1), where specific door opening frequency, $\lambda$, describes its ratio of open to closed in a given time interval.

$$
P(\text { open })=\lambda, P(\text { closed })=1-\lambda \quad \text { Eq. 4- } 1
$$

Due to lack of actual opening frequencies for the sample vessel, the opening frequencies of individual doors are based on door allowance category as derived in the EMSA III project (Jasionowski, 2015). The records do not provide one set of frequencies per category but permit the creation of proximate distributions for random sampling (Figure 4-5).

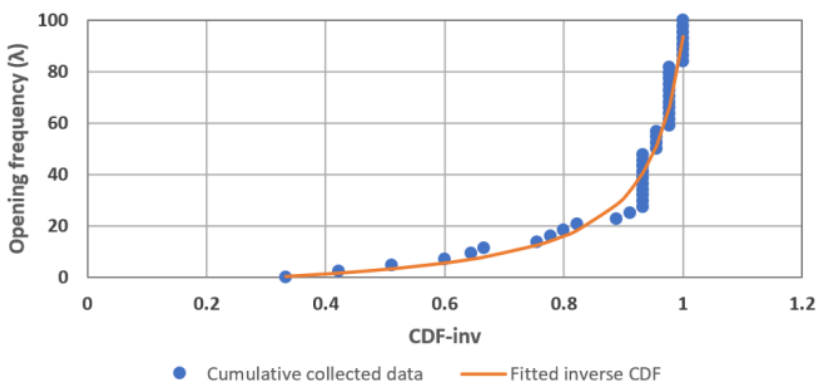

Figure 4-5: Proximate inverse cumulative density function for sampling of opening frequencies (category C-doors).

Each door in the sample vessel was assigned an opening frequency randomly sampled from a distribution derived for the corresponding opening allowance category. The randomly generated opening frequency for individual openings for the sample vessel is not listed here because of the large number of openings but Table 4-1 shows the expected (averaged) frequencies per door category.

\subsubsection{Opening leak and collapse}

Data regarding leaking and collapse pressure for a specific door may be obtained from the door manufacturer. However, SOLAS Reg. II-1/16.2 (IMO, 2006) specifies only minimum requirements for pressure testing of watertight doors, which would not provide a detailed measure of the actual collapse pressure. More detailed information regarding the leakage/collapse vulnerability of doors was assessed during the project FLOODSTAND (2012), where several full-scale model tests were performed to identify leak and collapse pressure heads for a range of opening categories. Detailed results are summarised in Jalonen et. al (2017). Possible variation in the leak and collapse parameters have also been studied in Ruponen (2017). However, in order to account properly for uncertainty related to values based on a small sample of doors only the uncertainty in collapse and leak pressure heights 
should be represented in a probabilistic framework by probability distributions for leakage and collapse pressure heads, as illustrated in Figure 4-6. In the figure, the collapse head is normally distributed around the specified mean collapse pressure, while the leak head is modelled by exponential distribution. Doors with higher leak resistance could also be modelled by a normal distribution.

Table 4-1: Assumed (expected) opening frequency for sample vessel opening types based on categories.

\begin{tabular}{|l|l|l|}
\hline Id. & Opening type, (Category) & $\lambda$ \\
\hline 1 & Sliding watertight door, (C) & 0.095 \\
\hline 2 & watertight hatch, (C) & 0.169 \\
\hline 3 & Hinged cold room door, (NA) & 0.044 \\
\hline 4 & Hinged provision room door, (NA) & 0.057 \\
\hline 5 & Sliding cold room doors, (NA) & 0.057 \\
\hline 6 & Sliding light- watertight door, (C) & 0.075 \\
\hline 7 & Sliding provision room door, (NA) & 0.055 \\
\hline 8 & Sliding semi- watertight door, (B) & 0.584 \\
\hline 9 & Escape hatch, (A) & 0.522 \\
\hline 10 & Hinged weathertight door, (A) & 0.032 \\
\hline 11 & Sliding weathertight door, (A) & 0.417 \\
\hline 12 & Hinged double fire door, (NA) & 0.690 \\
\hline 13 & B-class structure, (NA) & 0.000 \\
\hline 14 & Hinged escape door, (NA) & 0.564 \\
\hline 15 & Hinged non- watertight door, (NA) & 0.649 \\
\hline 16 & Sliding fire door, (NA) & 0.620 \\
\hline 17 & Hinged fire door, (NA) & 0.444 \\
\hline 18 & Sliding lift door, (NA) & 0.134 \\
\hline 19 & Hinged lift door, (NA) & 0.095 \\
\hline 20 & Unprotected doorway or conn., (NA) & 1.000 \\
\hline
\end{tabular}

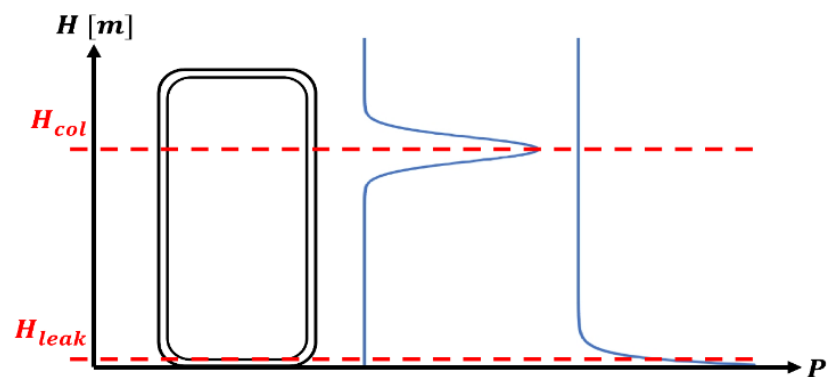

Figure 4-6: Door leak and collapse pressure heights modelled with probability distributions.

Recommended values for leakage area has been adopted from the FLOODSTAND project based on door category. Leakage through a gap at the bottom of doors (if present) is defined as a deterministic process governed solely by the position of the gap in relation to the floodwater level. For simplicity the pressure heads were assumed identical in both directions although doors (particularly hinged) may be characterised by different heads depending on the direction of the pressure gradient. Details on leak- and collapse pressure heads, including assumed distributions, are summarised in Table 4-2.

Table 4-2: Opening leak and collapse heads, including probabilistic models and parameters for various opening types for the sample vessel $(E(\lambda)$ and $N(\mu, \sigma)$ represent Exponential and Normal distribution).

\begin{tabular}{|c|c|c|c|c|c|}
\hline \multirow{2}{*}{ Id. } & Leak & Distribution & Col. & Distribution & Gap \\
\hline & [m] & $\mathbf{E}(\lambda) / \mathbf{N}(\mu, \sigma)$ & [m] & $\mathbf{N}(\mu, \sigma)$ & {$[-]$} \\
\hline 1 & NA & NA & 20.0 & $\mathrm{~N}($ Hcol, 0.1$)$ & NA \\
\hline 2 & NA & NA & 15.0 & $\mathrm{~N}($ Hcol, 0.1$)$ & NA \\
\hline 3 & 0 & $\mathrm{E}(100 / 3)$ & 3.50 & $\mathrm{~N}($ Hcol, 0.1$)$ & No \\
\hline 4 & 0 & $E(100 / 3)$ & 3.50 & $\mathrm{~N}($ Hcol, 0.1$)$ & No \\
\hline 5 & 0 & $\mathrm{E}(100 / 3)$ & 3.50 & $\mathrm{~N}($ Hcol, 0.1$)$ & No \\
\hline 6 & 2.5 & $\mathrm{~N}($ Hleak, 0.1) & 8.00 & $\mathrm{~N}(\mathrm{Hcol}, 0.1)$ & No \\
\hline 7 & 0 & $\mathrm{E}(100 / 3)$ & 3.50 & $\mathrm{~N}(\mathrm{Hcol}, 0.1)$ & No \\
\hline 8 & 2.5 & $\mathrm{~N}($ Hleak, 0.1) & 8.00 & $\mathrm{~N}($ Hcol, 0.1$)$ & No \\
\hline 9 & 0 & $\mathrm{E}(100 / 3)$ & 2.50 & $\mathrm{~N}($ Hcol, 0.1$)$ & No \\
\hline 10 & 0 & $E(100 / 3)$ & 2.50 & $\mathrm{~N}($ Hcol, 0.1$)$ & $\mathrm{No}$ \\
\hline 11 & 0 & $\mathrm{E}(100 / 3)$ & 1.00 & $\mathrm{~N}($ Hcol, 0.1$)$ & No \\
\hline 12 & 0 & $\mathrm{E}(100 / 3)$ & 2.00 & $\mathrm{~N}($ Hcol, 0.1$)$ & Yes \\
\hline 13 & 0 & $\mathrm{E}(100 / 3)$ & 1.50 & $\mathrm{~N}(\mathrm{Hcol}, 0.1)$ & No \\
\hline 14 & 0 & $\mathrm{E}(100 / 3)$ & 2.50 & $\mathrm{~N}($ Hcol, 0.1$)$ & Yes \\
\hline 15 & 0 & $\mathrm{E}(100 / 3)$ & 1.50 & $\mathrm{~N}($ Hcol, 0.1$)$ & No \\
\hline 16 & 0 & $\mathrm{E}(100 / 3)$ & 1.00 & $\mathrm{~N}($ Hcol, 0.1$)$ & Yes \\
\hline 17 & 0 & $\mathrm{E}(100 / 3)$ & 2.50 & $\mathrm{~N}(\mathrm{Hcol}, 0.1)$ & Yes \\
\hline 18 & 0 & $E(100 / 3)$ & 1.50 & $\mathrm{~N}(\mathrm{Hcol}, 0.1)$ & No \\
\hline 19 & 0 & $\mathrm{E}(100 / 3)$ & 1.50 & $\mathrm{~N}(\mathrm{Hcol}, 0.1)$ & No \\
\hline 20 & NA & NA & NA & NA & NA \\
\hline
\end{tabular}

\subsubsection{Loading condition}

The a-priori statistics for the sample vessel draught are available from its operational data but in the absence of actual data the distributions can be derived from statistics. Such approach was followed for the framework development and discrete draught distributions have been adopted from the research project eSAFE (Paterson \& Atzampos, 2017) and scaled to the operating range of the sample vessel, as illustrated in Fig. 5-8. A bounded Beta distribution, governed by Eq. 4-2, was fitted to the data. In the absence of actual operational data, the a-priori statistics for vertical centre of gravity, $K G$, are based on the stability booklet, although in actual operation the data would be available from the on-board loading computer. 

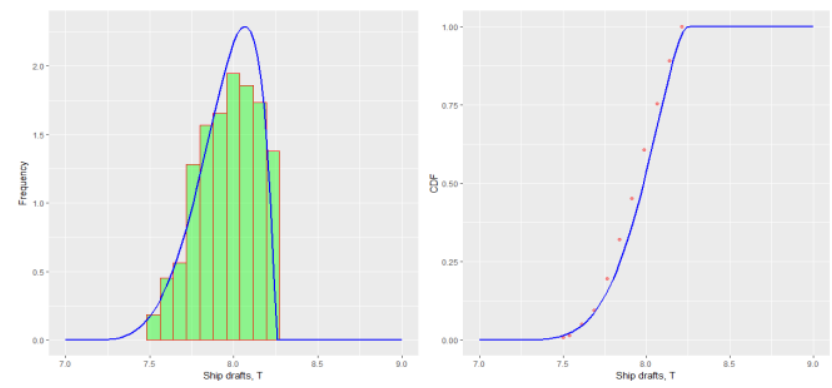

Figure 4-7: Numerical and analytical dimensionalised draft distribution as adopted from the eSAFE project.

$$
\begin{aligned}
& P(T)=\frac{(x-a)^{\alpha-1}(b-x)^{\beta-1}}{B(\alpha, \beta)(b-a)^{\alpha+\beta-1}} \quad \text { Eq. } 4-2 \\
& \alpha=5.2, \beta=1.8, a=7.1, b=8.25
\end{aligned}
$$

Nevertheless, the standard loading conditions from the stability booklet should reflect actual operating conditions of the vessel as illustrated in Figure 4-8. It is assumed that the loading conditions are normally distributed around the mean value represented by the dotted regression line. The orange lines correspond to $99 \%$ confidence bounds while the distribution is given by Eq. 4-3 to Eq. 4-5. As the mean value in Eq. 4-3 is represented by a linear function of the draught, $T$, given by Eq. 4-4, the distribution of $K G$ is conditional on draught $T$, denoted as $P(K G \mid T)$.

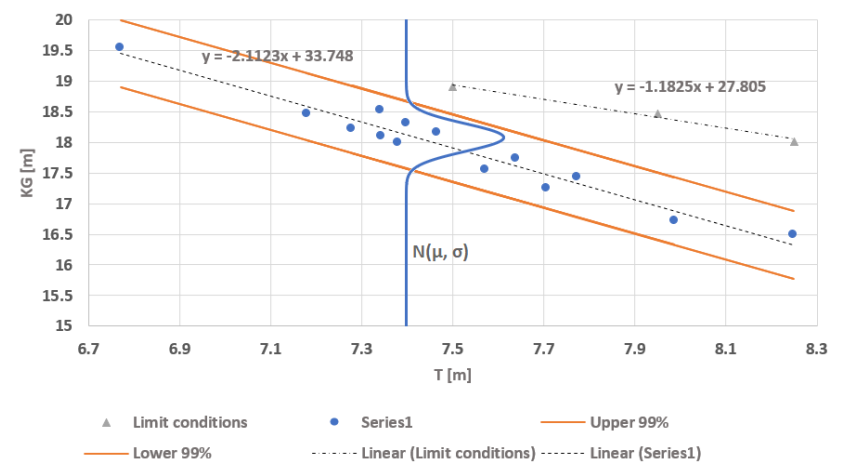

Figure 4-8: Operating loading conditions and assumed distribution.

This joint probability distribution can be derived with the help of the chain rule of conditional probability. A sample of 1,000 loading conditions drawn from the distribution shown in Figure 4-9, shows clearly that the vessel is operating in a narrow draught range biased towards the area of the summer load-line draught.

$$
P(K G \mid T)=\frac{1}{\sqrt{2 \pi \sigma^{2}}} e^{-\frac{(K G-\mu)^{2}}{2 \sigma^{2}}} \quad \text { Eq. 4-3 }
$$

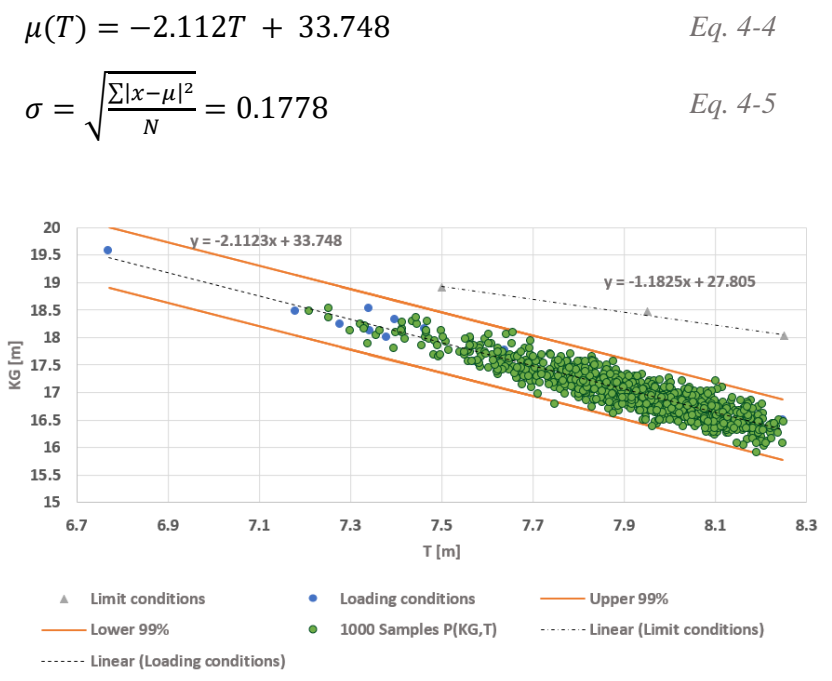

Figure 4-9: Samples of $K G$ and $T$ from developed distribution (the leftmost standard loading condition is the lightship condition, i.e. the area outside of the normal operational envelope).

\subsection{Probabilistic models}

\subsubsection{Door status sensor model}

The sample vessel has status sensors fitted to all watertight doors, providing this information to the operator. These doors can be closed remotely from the bridge. The likelihood function for the status sensor for doors should encode the probability of receiving a specific (either open $=1$ or closed $=0$ ) status from the sensor, $z$, conditional on actual door status, $x$. The likelihood function should therefore allow for four possible permutations of sensor and door statuses:

1. Sensor showing door open, provided that the door is open; $P(z=1 \mid x=1)$.

2. Sensor showing door closed, provided that the door is closed; $P(z=0 \mid x=0)$.

3. Sensor showing door open, provided that the door is closed; $P(z=1 \mid x=0)$.

4. Sensor showing door closed, provided that the door is open; $P(z=0 \mid x=1)$.

The first two combinations correspond to the intended function of the sensors. The remaining two represent false negatives and positive readings. Some sensor types have built-in error indication for this purpose, providing reliability figures for a specific sensor. Alternatively, these may be provided by the manufacturer. In this development, assumed sensor success rate for providing both open and closed indication is $99 \%$, which results in the probabilities 
(or likelihoods) presented in Table 4-3. The failure rate is heavily overestimated but from the lack of actual data, a conservative value was chosen. The success rates assume that the sensors are operational (i.e. not part of the damage breach). This relates directly to systems availability post damage and should be accounted for either by the sensor's reliability or by the direct update of the likelihood function. However, sensor availability was not implemented in the framework at this stage. As the above likelihoods encode discrete cases, the simplified normalisation constant $C$ cannot be applied, and the marginal probability distribution, $P(z)$ needs to be considered explicitly, as given in Eq. 4-6.

Table 4-3: Sensor likelihood, including sensor reliability (false positives/negatives).

\begin{tabular}{|l|l|l|}
\hline \multirow{2}{*}{ Sensor status, $\mathbf{z}$} & \multicolumn{2}{|l|}{ Actual door status, $\mathbf{x}$} \\
\cline { 2 - 3 } & $\boldsymbol{x}=\mathbf{1}$ (open) & $\boldsymbol{x}=\mathbf{0}$ (closed) \\
\hline$z=1$ & 0.99 & 0.01 \\
\hline$z=0$ & 0.01 & 0.99 \\
\hline
\end{tabular}

$$
P(x \mid z)=\frac{P(z \mid x) P(x)}{P(z)}=\frac{P(z \mid x) P(x)}{P(z \mid x) P(x)+P\left(z \mid x^{\prime}\right) P\left(x^{\prime}\right)} \quad \text { Eq. 4-6 }
$$

The posterior update may be illustrated by a simple example considering five doors with known apriori belief (opening frequencies) and specific sensor readings, $z$. Use of the likelihood functions derived above results in posterior probability updates as summarised in Table 4-4.

Table 4-4: Example of posterior update of door status probability using door sensor status.

\begin{tabular}{|l|l|l|l|l|}
\hline Door No. & $P(x=\mathbf{1})$ & $\boldsymbol{z}$ & $\boldsymbol{P}(\boldsymbol{Z}=\mathbf{z} \mid \boldsymbol{X}=\mathbf{1})$ & $\boldsymbol{P}(\boldsymbol{X}=\mathbf{1} \mid \boldsymbol{Z}=\mathbf{z})$ \\
\hline Door 1 & 0.50 & 0 & 0.01 & 0.010 \\
\hline Door 2 & 0.10 & 0 & 0.01 & 0.001 \\
\hline Door 3 & 0.05 & 1 & 0.99 & 0.839 \\
\hline Door 4 & 0.95 & 1 & 0.99 & 0.999 \\
\hline Door 5 & 0.20 & 0 & 0.01 & 0.003 \\
\hline
\end{tabular}

\subsection{Draught sensor model}

The sample vessel is fitted with four pneumatic draught sensors located on both sides at the forward and aft ends of the hull. The draught sensors readings enable continuous assessment of the floating position (expressed in terms of draught, heel and trim). The draught sensors may be used to construct a plane corresponding to the instantaneous waterplane of the ship. Mathematically, a plane is uniquely described by any three no-collinear points in space as shown given by Eq. 4-7.

$$
a\left(x-x_{0}\right)+b\left(y-y_{0}\right)+c\left(z-z_{0}\right)=0 \quad E q \cdot 4-7
$$

In the equation $a, b$ and $c$ are components of a vector $[a, b, c]^{T}$ normal to the plane while $\left(x_{0}, y_{0}, z_{0}\right)$ is an arbitrary point in the plane. The $x$ and $y$ are coordinates of the draught sensor in the body-fixed coordinate system and the $z$-coordinate the height of the water column at sensor location, as seen in Figure 4-10. The significance of the mathematical representation of the real-time waterplane is that it provides a reference to any arbitrarily chosen point within the vessel (e.g. an external or internal opening). This will form the basis for both predicting the flooding progression and the probabilistic modelling of flooding sensors, as discussed in the following.

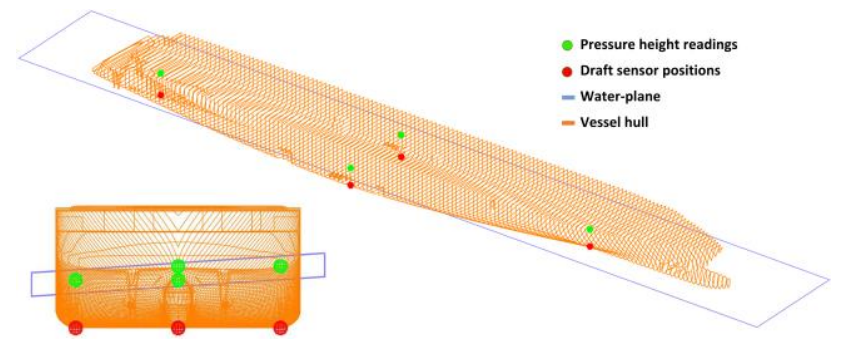

Figure 4-10: Mathematical representation of waterplane using draft sensor measurements.

\subsection{Initial flooding model}

The identified initial damage extents introduced in section 4.2.1 determine only whether given compartments are part of the breach. This, however, does not necessarily entail that they will be flooded when for example the breach (entire or in part) may be located above the waterplane. The probability of (initial) flooding is determined by three variables and their interplay: the lower limit of the vertical position of damage breach, $Z$, the draft of the intact vessel, $T$, and the significant wave height, $H_{s}$. For the purpose of the development, ship motions in waves were calculated by the time-domain simulation tool PROTEUS3 and the results of simulations were utilised to derive a probabilistic model representing initial flooding through the damage breach, conditional on the relevant variables $Z, T$, and $H_{s}$. The vessel $K G$ and wave encounter frequency (depending on speed and heading) would in fact also affect the 
initial flooding probability, but for simplicity these parameters were covered by the marginal a-priori distributions. In order to derive the distribution, 10,000 simulations were performed, with the significant wave height sampled from the interval $[0,15 \mathrm{~m}]$, heading from the interval $\left[0,360^{\circ}\right\rangle$ while draught, $T$, vertical centre of gravity, $K G$, and the waves zero up-crossing period, $T_{z}$, (conditional on the wave heights) were sampled from their marginal distributions presented in (DNV GL, 2017) for worldwide operation. Each time-domain simulation was run for 30 minutes. The results of simulations allow to determine maximum water elevation at random positions along the hull (where water ingress may be assumed if a breach were present). The water elevation is represented as the difference in height from the calm-waterplane represented by Eq. 4-8.

$$
\Delta Z=Z-T
$$

The values of $\Delta Z$ at which flooding occurs, conditional on $H_{s}$, are presented in Figure 4-11 indicating that the values of $\Delta Z$ are scattered and growing with increasing wave height. Flooding would, however, occur for all $\Delta Z$ values below this specific value, and the probability of flooding should be given by the exceedance probability. The data points are represented well by a Burr distribution, given by Eq. 4-9. The corresponding coefficients have been obtained by MLE and are presented as function of $H_{s}$, given by Eq. 4-10 to Eq. 4-12.

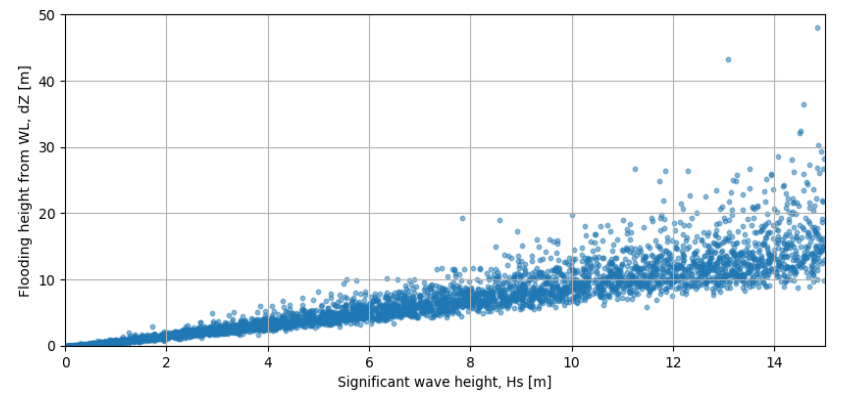

Figure 4-11: Height from calm-waterplane, $\Delta Z$, of initial flooding as a function of wave height, $H_{S}$.

$$
\begin{array}{ll}
\operatorname{PDF}\left(H_{s} \mid \Delta Z\right)=\frac{\alpha \beta}{\lambda}\left(\frac{\Delta Z}{\lambda}\right)^{\alpha-1}\left[1+\left(\frac{\Delta Z}{\lambda}\right)^{\alpha}\right]^{-\beta-1} & \text { Eq. 4-9 } \\
\alpha\left(H_{s}\right)=a+b e^{c H_{S}} & \text { Eq. 4-10 } \\
a=141.311, b=-141.309, c=-0.012 & \\
\beta\left(H_{S}\right)=a+b e^{c H_{S}} & \text { Eq. 4-11 }
\end{array}
$$

$$
\begin{array}{ll}
a=0.148, b=8.515, c=-0.422 & \\
\lambda\left(H_{s}\right)=a+b e^{c H_{s}} & \text { Eq. 4- } 12 \\
a=-28.634, b=29.753, c=0.020 &
\end{array}
$$

The cumulative (exceedance) probability may then be obtained by integrating Eq. 4-9, where the resulting Eq. 4-13 and Eq. 4-14 are valid on the intervals $[T, \infty]$ and $[-\infty, T\rangle$ respectively, as illustrated in Fig. 5-14.

$$
\begin{array}{ll}
P\left(\Delta Z \geq z \mid H_{s}\right)=\left[1+\left(\frac{\Delta Z}{\lambda}\right)^{\alpha}\right]^{-\beta}, Z \in[T, \infty] & \text { Eq. 4-13 } \\
P\left(\Delta Z \geq z \mid H_{s}\right)=1, \Delta Z \in[-\infty, T\rangle & \text { Eq. 4-14 }
\end{array}
$$

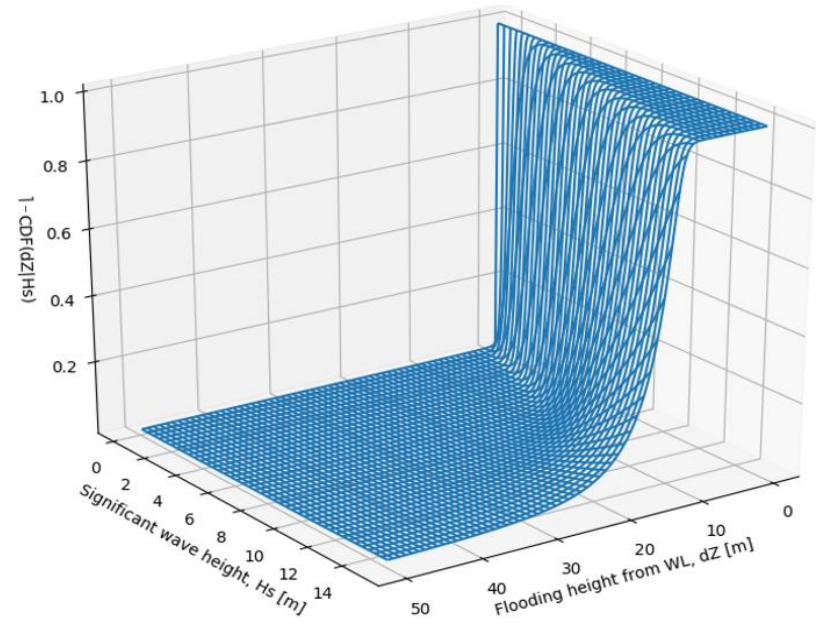

Figure 4-12: Exceedance probability of flooding height from $W L, \Delta Z$.

As the actual $\Delta Z$ value of the breach is not known, it must be assumed that the probability of flooding (conditioned on the initial damage extent) needs to be calculated for any of the $\Delta Z$ values within the interval $\left[\Delta Z_{1}, \Delta Z_{2}\right]$ (Figure 4-18). This can be accounted for by integrating Eq. 4-13 and Eq. 4-14 over the specific interval, which results in Eq. Eq. 4-15 to Eq. 4-18 and is illustrated in Figure 4-13.

$$
\begin{array}{ll}
P\left(H_{s} \mid \Delta Z_{1}, \Delta Z_{2}\right)=C\left(\Delta z_{2}{ }_{2} F_{1}\left(\frac{1}{\alpha}, \beta ; 1+\frac{1}{\alpha} ;-\left(\frac{\Delta z_{2}}{\lambda}\right)^{a}\right)+\cdots\right. & \text { Eq. 4-15 } \\
\left.\ldots-\Delta z_{1}{ }_{2} F_{1}\left(\frac{1}{\alpha}, \beta ; 1+\frac{1}{\alpha} ;-\left(\frac{\Delta z_{1}}{\lambda}\right)^{a}\right)\right), \quad \text { if } \Delta z_{1}>0 \text { and } \Delta z_{2}>0 \\
P\left(H_{s} \mid \Delta Z_{1}, \Delta Z_{2}\right)=C\left(\Delta z _ { 2 } F _ { 1 } \left(\frac{1}{\alpha}, \beta ; 1+\frac{1}{\alpha} ; \ldots\right.\right. & \text { Eq. 4-16 } \\
\left.\left.\ldots-\left(\frac{\Delta z_{2}}{\lambda}\right)^{a}\right)-\Delta z_{1}\right), \quad \text { if } \Delta z_{1}<0 & \text { Eq. 4-17 } \\
P\left(H_{s} \mid \Delta Z_{1}, \Delta Z_{2}\right)=C\left(\Delta z_{2}-\Delta z_{1}\right), \text { if } \Delta z_{1}<0 \text { and } \Delta z_{2}<0 & \text { Eq. 4-18 }
\end{array}
$$


As shown in the figure, Case 1 will flood regardless of the significant wave height because both vertical limits are located below the calm waterplane. Case 2, however, will not flood in calm water conditions as both integrands are located above the waterplane and the probability of flooding is increasing with wave height.

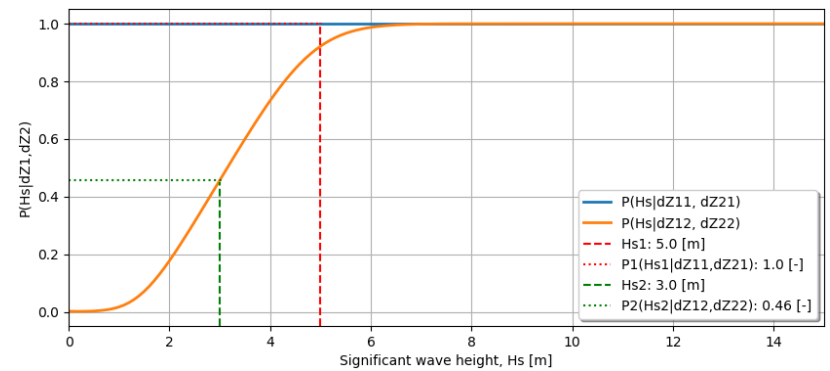

Figure 4-13: Example distribution for two initial damage extents (Case 1: integration over vertical interval $Z_{1.1}=1 \mathrm{~m}, Z_{2.1}=4 \mathrm{~m}$, significant wave height $H_{s .1}=5 \mathrm{~m}$ and draft $T_{1}=7.5 \mathrm{~m}$, and Case 2: integration over vertical interval $Z_{1.2}=9.0 \mathrm{~m}, Z_{2.2}=12.0 \mathrm{~m}$, significant wave height $H_{s .2}=3 \mathrm{~m}$ and draft $T_{1}=8 \mathrm{~m}$.).

\subsection{Progressive flooding model}

A probabilistic model of progressive flooding forms the basis for identifying possible progressive flooding extents for each of the initial extents identified in section 4.2.1. The model allows to estimate the probability of progressive flooding through an opening by taking into consideration the open/closed state of the openings, leak/collapse resistance and the position of the opening in relation to floodwater at any given time. Having a measure of the probability of progressive flooding through the individual openings, the Uncertain Graph Sampling (UGS) method as suggested by Karolius et al., (2019) may be utilised to populate the various progressive extent realisations by sampling. The conditions that will result in progressive flooding may be summarised as follows:

1. Opening has status open, has connection to initial damage extent and floodwater surface elevation, $W_{e}$ exceeds the lower vertical position of the opening, $Z$.

2. Opening has status closed, has connection to initial damage extent, leak head is smaller than collapse head producing substantial leak before collapse, and floodwater surface elevation, $W_{e}$ exceeds the opening leak pressure height, $H_{\text {leak }}$.
3. Opening has status closed, has connection to initial damage extent, with no substantial leak before collapse, and floodwater surface elevation, $W_{e}$ exceeds the collapse pressure height, $H_{c o l}$.

The above progressive flooding conditions (and the corresponding non-flooding conditions) may be illustrated as an event tree as shown in Figure 4-14. The condition that the respective openings must be connected to the flooding source is accounted for by the application of the UGS method separately and has not been represented in the event tree.

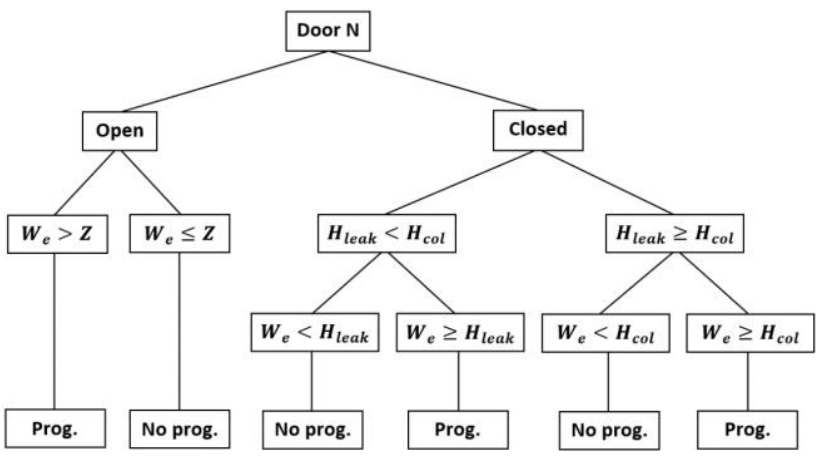

Figure 4-14: Event tree of progressive flooding realisations.

The closed status has two branches or sub-sets corresponding to the leak and collapse heads, giving the least resistance towards progressive flooding. Most openings will have a period of leakage before the pressure head is large enough to cause structural collapse (left branch of the closed status). The right branch shows leakage head higher than the collapse head, which is slightly misleading as this would never be the case. However, an opening may collapse without having a period of substantial leakage and is what this sub-set really represents. The event three can be used to derive a generic formula representing the progressive flooding probability as is given by Eq. 4-19 (representing the sum of all the subsets resulting in progressive flooding through an opening).

$$
\begin{aligned}
& P(\text { prog })=P(\text { open } \cap \text { prog })+P(\text { closed } \cap \text { prog }) \quad \text { Eq. } 4-19 \\
& =P(\text { open }) P\left(W_{e}>Z\right)+(1-P(\text { open }))\left[P\left(W_{e} \geq \min \left(H_{\text {leak }}, H_{\text {col }}\right)\right)\right]
\end{aligned}
$$

It is evident that the terms in in Eq. 4-19 representing the probability of particular open/closed status depend on opening frequency, $\lambda$, and can be modelled by a Bernoulli process. The remaining terms are all related to vertical positions and their distances to the floodwater elevation, either in the 
form of the lower limits of the openings or pressure heights. Regardless, they are all depending on the probability of the water elevation exceeding a certain vertical threshold and would therefore be well represented by the same probabilistic model. The probabilistic model for the exceedance probability of the floodwater elevation can be developed based on an approach similar to that for the initial flooding probability presented in section 4.5. The main distinction is that the vessel is no longer in intact condition and the water surface elevation of interest is that of the internal floodwater surface. In damaged ship conditions, 10,000 damage breaches were randomly sampled from the distributions discussed in section 4.2.1. Sampling of the vessel loading condition and wave related variables mirrored the process used for the initial flooding, while the door status as well as leak and collapse heads were randomly sampled from distributions introduced in section 4.2.3.

The 30 minutes-long time-domain simulations allowed to establish the maximum surface elevation within the flooded compartments providing the floodwater elevation with respect to the external calm-waterplane, $\Delta Z$. This, combined with the corresponding significant wave heights, enabled the development of a probabilistic model for floodwater exceedance for any compartment within the flooding extent, as a function of the significant wave height, $H_{s}$. The plot in Figure 4-15 shows the identified values of $\Delta Z$ at which floodwater elevation exceedance occurs plotted against the corresponding wave height, $H_{s}$. Similarly to the initial flooding probability, the variability of floodwater elevation is increasing with growing wave height. Some points, however, have negative values of $\Delta Z$ indicating the floodwater surface below the external waterplane (i.e. the water levels failing to equalise within the duration of the simulations). There are also some outliers representing vessel capsize (e.g. for wave height just above $H_{s}=3.0 \mathrm{~m}$ ), which data points align with the vertical axis as all originate from the same simulation case and represents individual flooded compartments. The data points are well represented by a GeneralisedLogistic probability distribution, as given by Eq. 420 .
The corresponding coefficients obtained by MLE, represented as a function of H_s are given by Eq. 422 to Eq. 424 . The probability of exceedance is obtained from the cumulative distribution as is represented by Eq. 421 and illustrated in Figure 416.

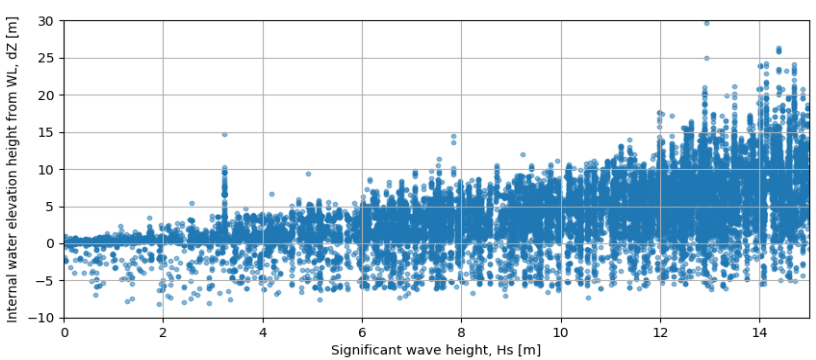

Figure 4-15: $\Delta Z$, between internal and external water elevation, as a function of wave height, Hs.

$$
\begin{aligned}
& P\left(\Delta Z \mid H_{S}, W_{e}\right)=-\left(\frac{\gamma}{\beta}\right)\left(e^{\left(\frac{\alpha-\Delta Z}{\beta}\right)}\right)\left(1+e^{\left(\frac{\alpha-\Delta Z}{\beta}\right)}\right)^{-\gamma-1} \quad \text { Eq. 4-20 } \\
& P\left(W_{e} \geq \Delta Z \mid H_{S}, W_{e}\right)=1-\frac{1}{\left(1+e^{\left(\frac{\Delta Z-\alpha}{\beta}\right)}\right)^{\gamma}} \quad \text { Eq. } 4-21 \\
& \alpha\left(H_{s}\right)=a+b e^{c H_{S}} \quad \text { Eq. 4-22 } \\
& a=-4.853, b=5.316, c=0.059 \\
& \beta\left(H_{s}\right)=a+b e^{c H_{s}} \quad \text { Eq. } 4-23 \\
& a=-26.546, b=26.138, c=-0.005 \\
& \gamma\left(H_{s}\right)=a+b e^{c H_{s}} \\
& a=0.096, b=0.382, c=0.065
\end{aligned}
$$

As we are interested in the exceedance probability of a vertical threshold, and not a vertical interval as was the case for the initial flooding probability presented above, Eq. 4-21 may be used directly without the need for integration. The final probabilistic model for progressive flooding is obtained by substituting Eq. 4-21 into Eq. 4-19 for the respective probability of exceedance terms. The door status probability is substituted by the Bernoulli process (Eq. 4-1), and the fact that the pressure heads are given as distributions are accounted for by integration, which results in Eq. 4-25. The $\Delta Z$ is now represented as the height from the external residual (post damage) waterplane, denoted as $W_{e}$. The representation of $\Delta Z$ for unprotected openings, and leak and collapse pressure heads for protected openings corrected for residual heel, $\varphi$, and trim, $\theta$, are given by Eq. 4-26 and Eq. 4-27. 


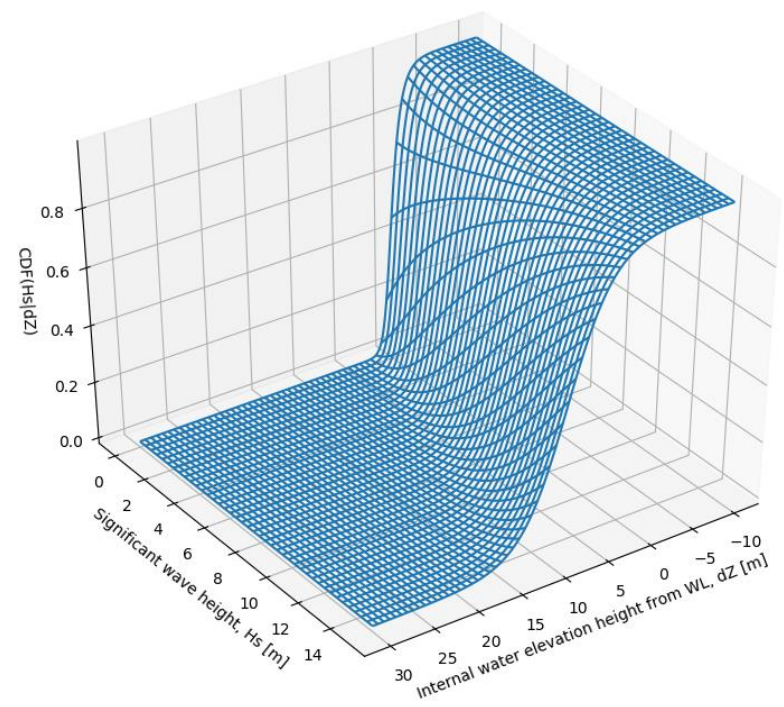

Figure 4-16: Exceedance probability for internal flooding height from the calm-waterplane.

$$
\begin{aligned}
& P(\operatorname{prog})=\lambda\left(1+e^{\left(\frac{\alpha-\Delta Z}{\beta}\right)}\right)^{-\gamma}+(1-\lambda) \iint_{Z_{\text {leak }}, Z_{\text {col }} \ldots} \ldots q .4-25 \\
& \ldots\left(1+e^{\left(\frac{\alpha-\min \left(Z_{\text {leak }}, Z_{\text {col }}\right)}{\beta}\right)}\right)^{-y} p d f\left(Z_{\text {leak }}, Z_{\text {col }}\right) d Z_{\text {leak }} d Z_{\text {col }}
\end{aligned}
$$

where, $p d f\left(Z_{\text {leak }}, Z_{\text {col }}\right)=p d f\left(Z_{\text {leak }}\right) p d f\left(Z_{\text {col }}\right)$, assuming independence.

$$
\begin{array}{ll}
\Delta Z=\left(Z-W_{e}\right) \cos (\varphi) \cos (\theta) & E q .4-26 \\
\Delta Z_{\text {col } / \text { leak }}=\left(Z-W_{e}\right) \cos (\varphi) \cos (\theta)+H_{\text {col } / \text { leak }} & E q .4-27
\end{array}
$$

\subsection{Flooding sensor model}

For the flooding sensors, the likelihood function should represent the probability of particular reading of a flooding sensor (positive or negative), given the specific total damage extent (initial and progressive). For that reason, the progressive extent realisations obtained from the UGS method with use of the progressive flooding probability model, developed in the foregoing, need to be combined with the corresponding initial damage extents. Therefore, the model for the flooding sensors needs to account only for the probability of water being actually present within the total extent in question, that it exceeds the vertical position of the sensor and whether the sensor is giving false positive readings or not. This can be achieved by adapting the probabilistic model developed for the exceedance probability introduced in the foregoing, combine it with the initial flooding probability and account for the sensor error rates. The model can again be represented as an event tree, as shown in Error! Reference source not found.

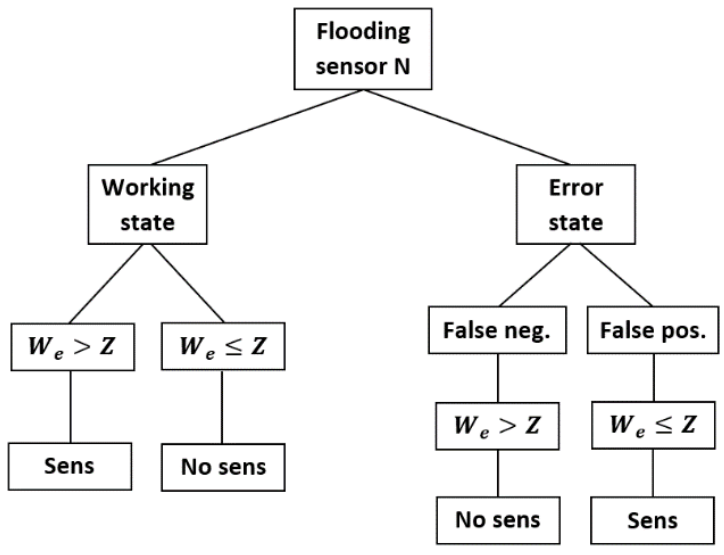

Figure 4-17: Event tree of flooding sensor status realisations.

The error state represents the proportion of time the sensor is not operational or provides incorrect readings, given by its rate of error, $\lambda_{e}$. The figure shows clearly that two sub-sets result in sensor status showing flooding, $z_{F}=1$, while two sub-sets result in sensor status showing no-flooding, $z_{F}=0$. The likelihood function for the flooding sensors is simply the summation of both sub-sets resulting in flooding status as is represented by Eq. 4-28, where $E_{s}$ and $W_{s}$ represent the error- and working-states, respectively. Substituting for the error rate, $\lambda_{\mathrm{e}}$, and the exceedance probability we obtain Eq. 4-29 and further simplification and rearranging in Eq. 4-30. Finally, by multiplying by the initial flooding probability, we obtain Eq. 4-31, which is the complete likelihood function for the flooding sensors for respective (complete) damage extents, where $x_{D}=\left[\Delta Z_{1}, \Delta Z_{2}\right]$, related to the initial extent. During the testing of the framework the error rate was assumed as $\lambda_{\mathrm{e}}=0.01$.

$$
\begin{array}{ll}
P\left(z_{F}=1 \mid x_{D}, H_{s}, W_{e}\right)=P\left(W_{s}\right) P\left(W_{e}>Z\right)+\cdots & \text { Eq. 4-28 } \\
\ldots+P\left(E_{s}\right) P\left(W_{e} \leq Z\right) & \\
=\left(1-\lambda_{e}\right)\left(1+e^{\left(\frac{\alpha-\Delta Z}{\beta}\right)}\right)^{-\gamma}+\cdots & \text { Eq. 4-29 } \\
\ldots+\lambda_{e}\left(1-\left(1+e^{\left(\frac{\alpha-\Delta Z}{\beta}\right)}\right)^{-\gamma}\right) & \\
=\left(1-2 \lambda_{e}\right)\left(1+e^{\left(\frac{\alpha-\Delta Z}{\beta}\right)}\right)^{-\gamma}+\lambda_{e} & \text { Eq. 4-30 } \\
\Lambda\left(\Delta Z_{1}, \Delta Z_{2}\right)=P\left(H_{s} \mid \Delta Z_{1}, \Delta Z_{2}\right) \ldots & \text { Eq. 4-31 } \\
\ldots\left[\left(1-2 \lambda_{e}\right)\left(1+e^{\left(\frac{\alpha-\Delta Z}{\beta}\right)}\right)^{-\gamma}+\lambda_{e}\right] &
\end{array}
$$

\subsection{AIS sensor model}

The breach size and the subsequent initial damage extent depend on the energy exchanged between the 
ships involved in the collision incident. The energy, in turn, is related to (among others) the size, speed and heading of the ships. In the following, an attempt is made to assess such relationships and utilise it for the development of likelihood functions. It is noteworthy that the available damage statistics have a very few data-points that include speed and heading of the striking vessels. This also applies to the size variables with the length of the striking vessel recorded most often and is therefore the parameter of choice for representing the size of the striking vessels. The breach variables influenced by the available energy are: vertical position, $Z$, length, $L$, height, $H$, and transverse penetration, $Y$, while the longitudinal position, $X$, relates to the heading at the instant of collision and is assumed to be independent on the striking vessel size. The likelihood function for AIS data should represent the probability of receiving information of a specific vessel length from the AIS receiver, conditional on the respective damage variables, and is given by the conditional distribution of the vessel length and the respective damage variables.

The relevant distributions from available accident statistics discussed in section 4.2.1 and presented in detail in (Karolius, 2019) may be utilised for this purpose. It is noteworthy, however, that the striking vessel size is marginalised from these distributions. Nevertheless, since the actual size could be known from AIS data the distributions can be given as conditional on the breach parameters represented as a function of vessel length. Detailed scatter plots presented in (Karolius, 2019) show an increase in the variability of the data points with increasing striking vessel length. This indicates that the breach variables are mainly located in the lower regions for smaller striking vessel lengths, and as the striking vessel length increases, the valid range of breach variables is expanding to also include the upper region. This suggests that smaller breach sizes are also viable for larger striking vessel lengths and is presumably a consequence of not accounting for the speed nor heading. On the other hand, it is clearly unlikely that short (i.e. small) striking ships may cause very large breaches. The observed growing variability (standard deviation) allows for constructing conditional distributions by representing the respective distribution coefficients as functions of the striking vessel length, and fitting new coefficients using MLE, as summarised in (Karolius, 2019).

As for the model for the initial flooding probability presented above, the discrete initial damage extents are of interest rather than continuous breach variables, and the distributions need to be integrated over the relevant intervals related to specific initial damage extents (or damage cases) comprising the integration domain. For highly complex subdivisions, identifying the relevant intervals for the integration domain (i.e., the upper and lower bounds) may prove difficult and time consuming. An alternative method involves identifying the domain by random sampling. In this approach the bounds are associated with extreme coordinates of the sample point lying within the specific extent. The method is also time-consuming but much simpler than identification by the compartment extents. Furthermore, the time overheads are not of prime importance as the process is executed only once. Example of integrand intervals are illustrated in Figure 4-18 for the respective variables while the resulting likelihood functions after integration are given by Eq. 4-32 to Eq. 4-35. The function ${ }_{2} F_{1}(a, b ; c ; z)$ in Eq. $4-34$ is the Hypergeometric function.

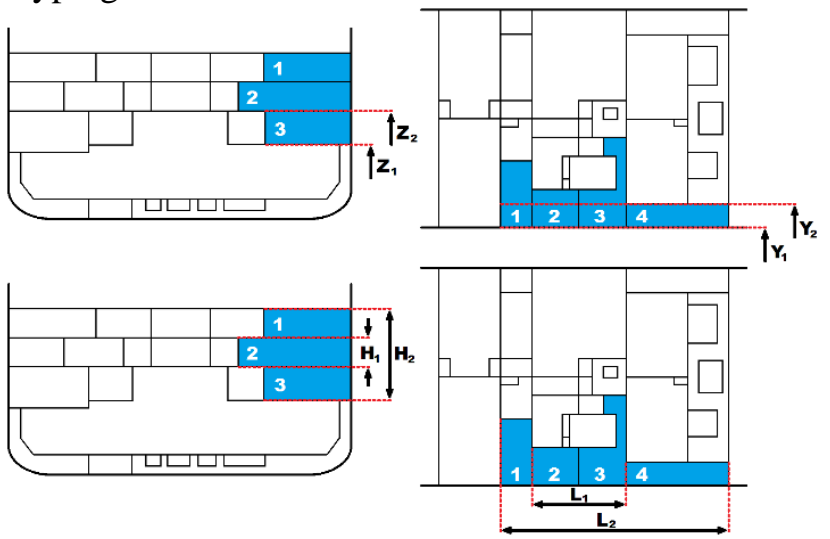

Figure 4-18: Example of integrand intervals for the respective breach variables for specific initial damage extents (right case with three damage comp. differ from the left case with four damaged comp).

$$
\begin{aligned}
& \operatorname{PDF}\left(L_{B P} \mid Z_{1}, Z_{1}, T\right)=\frac{1}{2\left(Z_{1}-Z_{2}\right)} \sinh \left(\frac{Z_{1}-Z_{2}}{2 s}\right) \ldots \\
& \ldots \operatorname{sech}\left(\frac{m+T-Z_{1}}{2 s}\right) \operatorname{sech}\left(\frac{m+T-Z_{2}}{2 s}\right) \\
& \text { where; } s\left(L_{B P}\right)=a L_{B P}+b, a=0.0209, b=0.0030, \\
& m\left(L_{B P}\right)=c L_{B P}+d, c=-0.0035, d=-1.2329 \\
& P D F\left(L_{B P} \mid L_{1}, L_{1}\right)=\frac{1}{L_{2}-L_{1}}\left(\left(1+\left(r L_{1}\right)^{b}\right)^{-a}+\cdots\right.
\end{aligned}
$$




$$
\begin{aligned}
& \left.\ldots-\left(1+\left(r L_{2}\right)^{b}\right)^{-a}\right) \\
& \text { Where; } a=5.9883, b=0.9576, \\
& r\left(L_{B P}\right)=c+d e^{e L_{B P}, c=0.0185, d=0.3015, e=-0.0420} \\
& P D F\left(L_{B P} \mid H_{1}, H_{1}\right)=\frac{\left(\frac{H_{2}}{b}\right)^{a p}{ }_{2 F_{1}}\left(p, p+q ; p+1 ;-\left(\frac{H_{2}}{b}\right)^{a}\right)}{\left(H_{2}-H_{1}\right) p B(p, q)}+\cdots \quad \text { Eq. 4-34 } \\
& \quad \ldots-\frac{\left(\frac{H_{1}}{b}\right)^{a p}{ }_{2} F_{1}\left(p, p+q ; p+1 ;-\left(\frac{H_{1}}{b}\right)^{a}\right)}{\left(H_{2}-H_{1}\right) p B(p, q)} \\
& \text { Where; } a=6.8089, p=0.08383, q=1.1089, \\
& b\left(L_{B P}\right)=b+c e^{d L_{B P}, b=-121.9882, c=122.0882, d=0.0010} \\
& \text { PDF }\left(L_{B P} \mid Y_{1}, Y_{1}\right)=\frac{1}{Y_{2}-Y_{1}}\left(e^{-\lambda Y_{1}}-e^{-\lambda Y_{2}}\right) \\
& \text { Where; } \lambda\left(L_{B P}\right)=a+b e^{c L_{B P},} \\
& a=0.2524, b=29.7476, c=-0.0883
\end{aligned}
$$

\section{COMPLETE FUSION FRAMEWORK}

In the foregoing sections, comprehensive probabilistic models have been developed for use within a multi-sensor fusion methodology. In the following, their combined implementation as a complete framework will briefly be discussed and reviewed. The review will be supported by the schematic layout of the methodology illustrated in Appendix I. The main variable of interest, $X$, is the damage extent and location. This is represented by a set of compartments within the damage breach (initial extent, $i$ ), which may be connected to additional compartments through the internal subdivision boundaries (progressive extent, $j$ ). The complete set of compartments therefore comprises two disjoint subsets, $x_{i}, x_{j}$, as represented by Eq. 5-1 to Eq. 5-3.

$$
\begin{array}{ll}
X\left(x_{i}, x_{j}\right)=\left[x_{i}, x_{j}\right] & E q .5-1 \\
x_{i}=\left[\operatorname{comp}_{i .1}, \operatorname{comp}_{i .2}, \ldots, \operatorname{comp}_{i . N_{i}}\right] & E q .5-2 \\
x_{j}=\left[\operatorname{comp}_{j .1}, \operatorname{comp}_{j .2}, \ldots, \text { comp }_{j . N_{j}}\right] & \text { Eq. 5-3 }
\end{array}
$$

Initial screening by the fusion process is based on the evidence from draught sensors and AIS. This is done only once and at the time instant time $t_{0}$ immediately before the collision as is seen at the bottom of Figure I-1 in Appendix I. Since this initial update is non-recursive, Eq. 3-11 may be used directly, combining the a priori list of initial damage extents, as obtained in section 4.2.1 (subset $x_{i}$ ) with the AIS likelihood functions; Eq. 4-32 to Eq. 4-35.

The next fusion process is done recursively for each time, $t_{k}=t_{k-1}+\Delta t$, at fixed time-steps, $\Delta t$.
Initially, the posterior update of the opening probabilities combines the a priori opening frequencies with the sensor readings for opening status using Eq. 4-6. This must be part of the recursive process, as doors may change status. The updated posterior opening probabilities are then merged with the a priori leak- and collapse model (from section 4.2.3), and in combination with the currently observed wave height and floating position (from section 4.4), it forms the progressive flooding probabilities for the respective openings, using Eq. 4-25. Possible realisations of progressive extents (subset $x_{i}$ ) are sampled with the help of the Uncertain Graph Sampling (UGS) method for each of the initial damage extents.

The combined sets ( $i$ and $j$ ), represent the complete samples space of possible flooding realisations, $X\left(x_{i}, x_{j}\right)$, with each of the samples assigned a new posterior probability of occurrence. Once all the flooding realisations are established the flooding sensor readings can be utilised to provide the final update at this time-step. This is done using Eq. 4-31 in combination with the damage extent probabilities and Eq. 3-12. The final posterior probability acts as the a priori for the next time step. As the UGS method is applied for every time-step, the various sampled progressive extents may differ between the time-steps simply because new evidence might be made available (e.g. change in residual draft). The a priori for the next time-step should therefore be in the form of the initial extents alone (subset $i$ ). This is obtained from the summation of all the $\mathrm{n}$ progressive subsets $j$ originating from $i$, represented by Eq. 5-4.

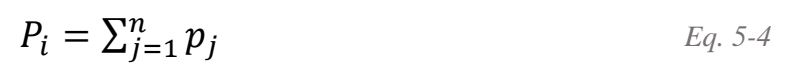

\section{IMPLEMENTATION AND TESTING}

All the probabilistic models introduced in the foregoing have been combined and implemented as a demonstration platform for application within flooding emergency scenarios. The platform reads emulated sensor data (for this purpose time series from PROTEUS3 simulations are interpreted as actual sensor readings) and provides sequential updates based on the methodology described in the 
previous sections. The presented results follow the successive updates triggered by new sensor evidence to improve the accuracy of flooding prediction, and to reflect the ensuing reduction in uncertainty for each implemented update. The posterior updates from the flooding sensors are by the demonstration platform executed every 5 minutes and accounted for by Eq. 3-12 (six successive updates at 5, 10, 15, 20, 25 and 30 minutes executed within 30 minutes of timedomain simulations).

\subsection{Test scenarios}

To illustrate the application of the framework within realistic flooding emergency scenarios, Karolius (2019) presents a range of test-scenarios, with various combinations of striking vessel length and sea states. In the following, however, only two selected cases are included for illustration, corresponding to striking vessel lengths of 100 and 250 meters, respectively. Both scenarios have been applied in two different wave conditions - calmwater, and extreme wave height $\left(H_{s}=10.0 \mathrm{~m}\right)$. The corresponding damage breaches have been sampled from the distributions discussed in section 4.8, conditioned on the striking vessel's length, and relevant dependencies between the individual dimensions of the breach where given by the distributions discussed in section 4.2.1.

Most variables have in fact been obtained by random sampling for a realistic representation, i.e. they are unknown and inferred using the presented framework. The stricken vessel loading condition, represented by the draft, $T$, and the vertical centre of gravity, $K G$, were sampled from the distributions developed in section 4.2.4, assuming that the vessel operates at even keel $\operatorname{Tr}=0$. The vessel's heading was sampled uniformly on the interval $\left[0,360^{\circ}\right\rangle$, and the openings' status, and leak- and collapse heads were sampled from the distributions developed in section 4.2.3. The two test-scenarios and corresponding variables are summarised in Table 6-1. The ship model selected for implementation and testing is based on a large (100,000 GT) modern cruise vessel currently in operation. Main particulars of the vessel are presented in Table 6-2.
Table 6-1: Summary of test-scenarios: Striking-vessel length (top two rows), stricken vessel loading condition, resulting breach and wave environment.

\begin{tabular}{|l|l|l|}
\hline Striking vessel & V1 & V2 \\
\hline Length, $L_{B P}[\mathrm{~m}]$ & 100.00 & 250.00 \\
\hline Stricken vessel & L1 & L2 \\
\hline Draft, $T[\mathrm{~m}]$ & 7.72 & 8.13 \\
\hline VCG $/ K G[\mathrm{~m}]$ & 17.51 & 16.71 \\
\hline Trim, $\operatorname{Tr}[\mathrm{m}]$ & 0.00 & 0.00 \\
\hline Breach boundary & $\mathbf{B 1}$ & $\mathbf{B 2}$ \\
\hline Longitudinal position, $X[\mathrm{~m}]$ & 103.1 & 145.3 \\
\hline Vertical position, $Z[\mathrm{~m}]$ & 6.23 & 2.40 \\
\hline Length, $L[\mathrm{~m}]$ & 1.73 & 53.49 \\
\hline Height, $H[\mathrm{~m}]$ & 1.89 & 5.16 \\
\hline Penetration, $Y[\mathrm{~m}]$ & 0.64 & 1.20 \\
\hline Number of zones (initial) & 1 & 4 \\
\hline Number of comp. (initial) & 1 & 6 \\
\hline Wave environment & $\mathbf{W 1}$ & $\mathbf{W 2}$ \\
\hline Heading, $H_{g}\left[{ }^{\circ}\right]$ & 94.23 & 349.99 \\
\hline Wave height $H_{s}[\mathrm{~m}]$ & $0.0,10.0$ & $0.0,10.0$ \\
\hline
\end{tabular}

Table 6-2: Particulars of the sample ship.

\begin{tabular}{|l|l|}
\hline Parameter (symbol) & Value [designation] \\
\hline Length between perp. $\left(L_{B P}\right)$ & $273.00[\mathrm{~m}]$ \\
\hline Breadth $(B)$ & $36.00[\mathrm{~m}]$ \\
\hline Depth $(D)$ & $21.00[\mathrm{~m}]$ \\
\hline Gross tonnes $(G T)$ & $100000.00[-]$ \\
\hline Number of passengers $(-)$ & $2800[$ persons] \\
\hline Number of crew $(-)$ & 1050 [persons] \\
\hline
\end{tabular}

The vessel internal compartment connectivity comprises a total of 894 openings, covering doors, hatches, etc. The model of the internal arrangement is shown in Figure 6-1. The flooding detection system comprises a total of 52 pneumatic and 94 level sensors fitted in dry spaces and tanks, respectively. The sensor arrangement complies with the IMO regulations as set out in guidance note MSC.1/Circ.1291 (IMO, 2008), requires the flooding sensors to be fitted in compartments exceeding a certain size limit (i.e. those that either have a volume in cubic metres larger than the vessel moulded displacement per centimetre immersion at deepest subdivision draught or have a volume in excess of 30 cubic metres). The vessel has four draft sensors: one fore, one aft, and two at midship, located at either side. Door status sensor are fitted on all doors classified as watertight (power operated-, semi-, and light-watertight doors). 


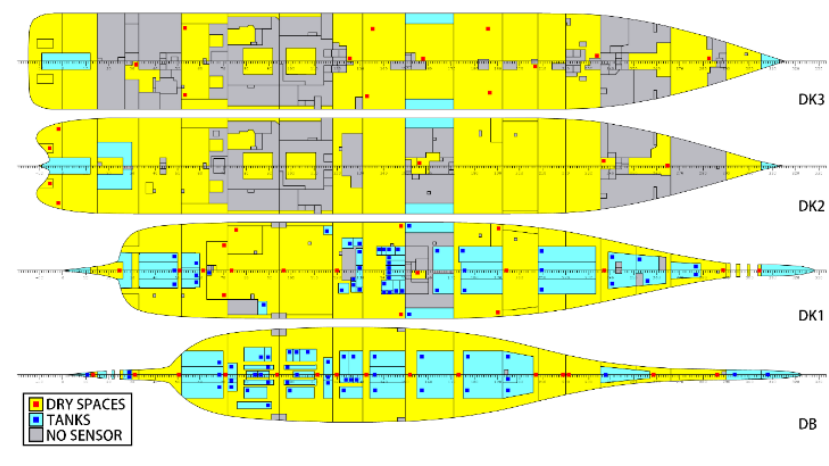

Figure 6-1: Arrangement of flooding sensors in dry and liquid spaces.

\subsection{Presentation}

To limit the extent, only selected result tables has been included. For more comprehensive results, reference is made to (Karolius, 2019). In the following, the main findings are summarised and discussed using the ten most likely unique damage extents (unique combination of initial and progressive extent represented by $\left.X\left(x_{i}, x_{j}\right)\right)$ inferred by the fusion process. The successive reduction in uncertainty is measured by the number of cases constituting the 99\% Confidence Interval (CI) (i.e. number of cases with aggregated total probability of $99 \%$ ). The actual damage extent is presented graphically on deck plans with a dark blue colour representing the initial extent and the successive progressive extents shown in a lighter shade of blue. Green markers denote the locations of flooding sensors relevant to the test-cases and the damage breach is shown in red.

\subsection{Implementation and testing result}

\subsubsection{No sensor evidence}

As discussed in section 4.2.1 the MC sampling produced 19,255 unique initial damage extents. From these, 10,915 is represented by the $99 \%$ CI seen in Table 6-3. That is, without any sensor evidence, prediction with $99 \%$ confidence results in 10,915 possible initial flooding extents. Furthermore, the distribution of damage cases is biased towards single compartment damages located in the fore region of the vessel in line with the distributions discussed in section 4.2.1. In the absence of sensor evidence the predicted a-priori initial extents are identical for both test cases. The subsequent updates following the sensor reading and unique for each test-case, are summarised and discussed in the following two sections.
Table 6-3: No sensor evidence.

\begin{tabular}{|c|c|c|c|}
\hline Damage: & Probability: & Compartments: & \\
\hline DAM6 6 & 0.0842 & R180001 & \\
\hline DAM1 & 0.0305 & R180001, R180002 & \\
\hline DAM13 & 0.0247 & R180001, R180401 & \\
\hline DAM17 & 0.0234 & R170101 & \\
\hline DAM65 & 0.0139 & R180001, R180002, R180401 & \\
\hline DAM29 & 0.0137 & R180001, R170101 & \\
\hline DAM46 & 0.0105 & R040112 & \\
\hline DAM2 & 0.0090 & R180001, R170001, R170101 & \\
\hline DAM14 & 0.0085 & R100108 & \\
\hline DAM147 & 0.0084 & R090113 & \\
\hline $100 \% \mathrm{CI}$ & $99 \% \mathrm{CI}$ & $95 \% \mathrm{CI}$ & $90 \% \mathrm{CI}$ \\
\hline 19225 & 10915 & 3057 & 1499 \\
\hline
\end{tabular}

\subsubsection{Test-case 1 -Calm-water}

Test-case 1, involving striking ship of $100 \mathrm{~m}$ length, is illustrated in Figure 6-2, showing one initially breached compartment (R070101) and three progressively flooded compartments (R070201, EX070101, R070102).

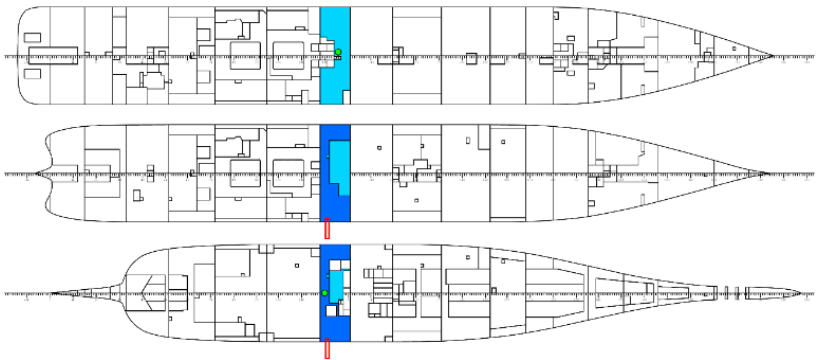

Figure 6-2: Test-case 1, Striking vessel length: $100.0 \mathrm{~m}, \mathrm{H}_{\mathrm{S}}=0.0 \mathrm{~m}$.

The posterior update from AIS and draft sensors sees the actual case DAM421 ranked $8^{\text {th }}$ (Table 6-4). The update provides a reduction in number of cases representing the $99 \%$ CI from 10,915 to 3,062 cases, a reduction that comes from a shift in focus from larger to smaller extents due to the available evidence of relatively small size of the striking ship. Probability of extreme damage extents are subsequently reduced, while smaller, more likely extents, increased and in line with the distributions discussed in section 4.8. The results incorporated with evidence from flooding sensors for the calm water case at time step $t=$ $5.0 \mathrm{~min}$ (Table 6-5) indicate that the prediction is rapidly closing in towards the actual damaged region, showing that various progressive extent realisations of the actual damage DAM421 are dominating the ten most likely cases, with the actual case ranked fourth with a probability of 0.05 . Top-ranked, with probability 0.4 , is a realisation of the actual case but without compartment R070201 in the progressive extent. This indicates that the prediction is in fact targeting the damaged region, just not ranking the actual unique case on top. Compartment R070201 has 
a flooding sensor fitted but it does not indicate flooding because ingress is slow, originating from leakage through an escape hatch with the floodwater level yet to reach the sensor.

Table 6-4: Inference from draft and AIS sensor (actual case in red).

\begin{tabular}{|c|c|c|c|}
\hline \multicolumn{4}{|c|}{ Predicted initial extent } \\
\hline Damage: & Probability: & & Compartments: \\
\hline DAM13 & 0.0328 & & R180001, R180401 \\
\hline DAM6 & 0.0232 & & R180001 \\
\hline DAM1 & 0.0220 & & R180001, R180002 \\
\hline DAM17 & 0.0217 & & R170101 \\
\hline DAM65 & 0.0129 & & R180001, R180002, R180401 \\
\hline DAM345 & 0.0121 & & R170101, R170307 \\
\hline DAM107 & 0.0097 & & R090113, R090305 \\
\hline DAM421 & 0.0094 & & R070101 \\
\hline DAM24 & 0.0088 & & R090113, R090305, R090401 \\
\hline DAM519 & 0.0081 & & R170101, R170302, R170308 \\
\hline $\begin{array}{c}\mathbf{1 0 0} \% \text { CI: } \\
19225\end{array}$ & $\begin{array}{c}99 \% \mathrm{CI}: \\
3062\end{array}$ & $\begin{array}{c}\text { 95\% CI: } \\
1223\end{array}$ & $\begin{array}{c}\text { Case rank.: } \\
8 \\
\end{array}$ \\
\hline \multicolumn{4}{|c|}{ Actual initial extent: } \\
\hline & & & $\begin{array}{c}\text { Compartments: } \\
\text { R070101 }\end{array}$ \\
\hline
\end{tabular}

Table 6-5: Inference from flooding sensors, $H_{S}=0 \mathrm{~m}, t=5 \mathrm{~min}$.

\begin{tabular}{|c|c|c|c|c|c|}
\hline \multicolumn{6}{|c|}{ Predicted (complete damage) extent } \\
\hline \multirow{2}{*}{ Dam: } & \multirow{2}{*}{ Prob.: } & \multicolumn{3}{|c|}{ Compartments: } & \\
\hline & & \multirow{2}{*}{$\begin{array}{l}\text { Initial: } \\
\text { R070101 }\end{array}$} & \multicolumn{3}{|l|}{ Progressive: } \\
\hline DAM421 & 0.4041 & & \multicolumn{3}{|c|}{ EX070101, R070102 } \\
\hline DAM1506 & 0.0670 & R070101, R070204 & \multicolumn{3}{|c|}{ EX070101, R070102 } \\
\hline DAM421 & 0.0588 & R070101 & \multicolumn{3}{|c|}{ EX070101, R070102, R070203, R070201 } \\
\hline DAM421 & 0.0540 & R070101 & \multicolumn{3}{|c|}{ EX070101, R070102, R070201 } \\
\hline DAM421 & 0.0309 & R070101 & \multicolumn{3}{|c|}{ R070201, R070202, R070203, R070205, R070102, EX } \\
\hline DAM421 & 0.0270 & R070101 & \multicolumn{3}{|c|}{ R070201, R070202, R070205, R070102, EX070101 } \\
\hline DAM421 & 0.0266 & R070101 & \multicolumn{3}{|c|}{ EX070101, R070102, R070205, R070201 } \\
\hline DAM421 & 0.0266 & R070101 & \multicolumn{3}{|c|}{ R070201, R070203, R070205, R070102, EX070101 } \\
\hline DAM40 & 0.0193 & R070101, R070201 & \multicolumn{3}{|c|}{ EX070101, R070203, R070102 } \\
\hline DAM40 & 0.0172 & R070101, R070201 & \multicolumn{3}{|c|}{ EX070101, R070102 } \\
\hline \multicolumn{2}{|c|}{421153} & $\begin{array}{c}99 \% \mathrm{Cl}: \\
81\end{array}$ & $\begin{array}{c}95 \% \text { CI: } \\
49\end{array}$ & $\begin{array}{c}90 \% \text { CI: } \\
33\end{array}$ & $\begin{array}{c}\text { Case rank.: } \\
4\end{array}$ \\
\hline Positive & ensor rea & ding in: & & t no reading: & R070201 \\
\hline \multicolumn{6}{|c|}{ Actual (complete damage) extent: } \\
\hline \multicolumn{2}{|l|}{ Name: } & \multirow{2}{*}{$\begin{array}{l}\text { Initial: } \\
\text { R070101 }\end{array}$} & \multicolumn{2}{|c|}{ Progressive: } & \\
\hline DAM421 & & & R0702 & 101, R070102 & \\
\hline
\end{tabular}

Table 6-6: Inference from flooding sensors, $H_{S}=0 \mathrm{~m}, t=30 \mathrm{~min}$.

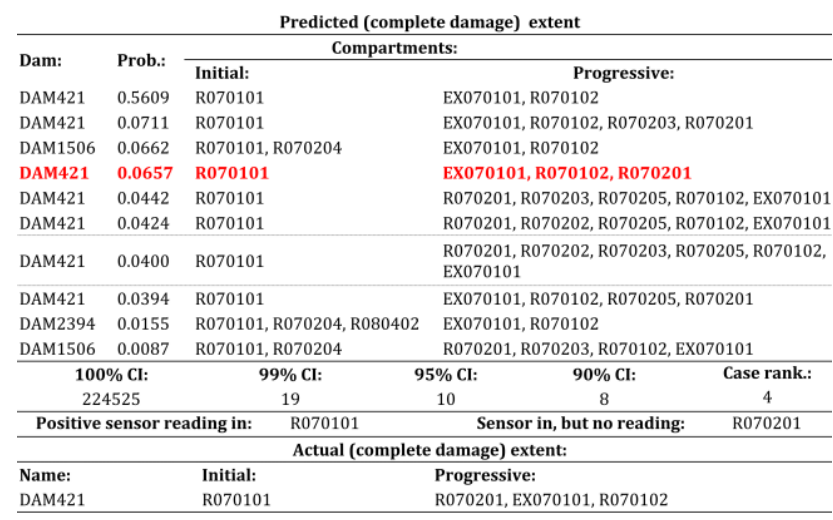

As a matter of fact, the sensor will not submerge within the 30-minute simulation time. As a result, the likelihood function assigns lower probability to the actual case than to the realisations without R070201, simply because it is more likely. At the last time step, $t=30 \mathrm{~min}$ (Table 6-6), the actual case has a probability of 0.07 and ranks $4^{\text {th }}$ while the top-ranked case (without R070201) has a probability of 0.56 . The 99\% CI comprises 19 cases, down from 81 cases at the initial time-step.An alternative way in presenting the result will also be used where flooding probability is assigned to respective compartments by adding contributions of probabilities from individual damage cases from a specific $\mathrm{CI}$. This enables presenting the most likely compartments being flooded independently with a specific confidence by utilising colour coding, or heat maps as is seen in Figure 6-3 for the last time step of the calm water case. The alternative presentation method has a significant advantage over the ranked list of unique (initial- and progressive) extents, as an important parameter affecting the predictions is the sensor density. Many of the compartments (smaller compartments) doesn't have flooding sensors, making it difficult for the framework to distinguish between the unique flooding cases. The colours are representing the probability, where red correspond to a probability of one, yellow to a probability of 0.01 and green to a probability of zero, i.e. linearly mapped to an RGB colour profile. The graphical representation clearly provide significant supportive information to the crew, regardless of the actual (unique) case not being ranked on top.

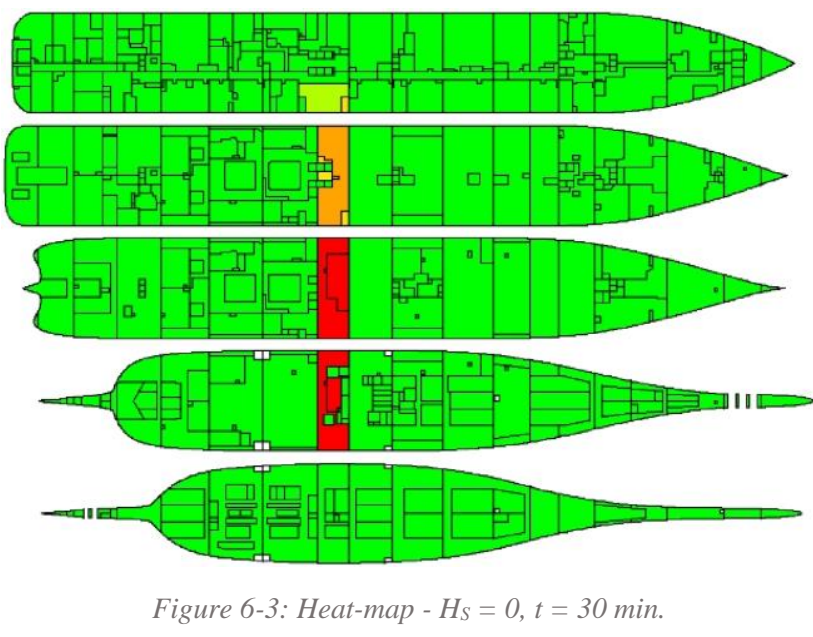

\subsubsection{Test-case 1 - Waves}

For the wave case, the sensor in R070201 is also here not showing indication (Table 6-7) where the actual case is ranked $29^{\text {th }}$. This is, however, only in the first two time-steps as the wave-induced motions enhance the leakage through the opening, submerging the sensor and providing flooding indication for the remaining time-steps. The probability of the actual case is therefore, for $t=15 \mathrm{~min}$, raised to a value of 
0.13 (Table 6-8). In the last few time steps, however, progressive extent realisations with even more compartments (without sensors) are assigned higher probabilities (Table 6-9).

Table 6-7: Inference from flooding sensors, $H_{S}=10 \mathrm{~m}, t=5 \mathrm{~min}$.

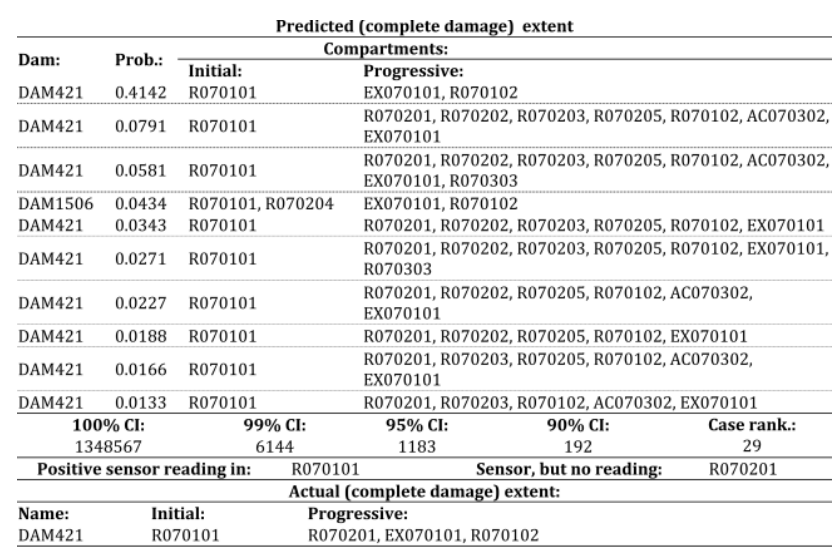

Table 6-8: Inference from flooding sensors, $H_{S}=10 \mathrm{~m}, t=15 \mathrm{~min}$.

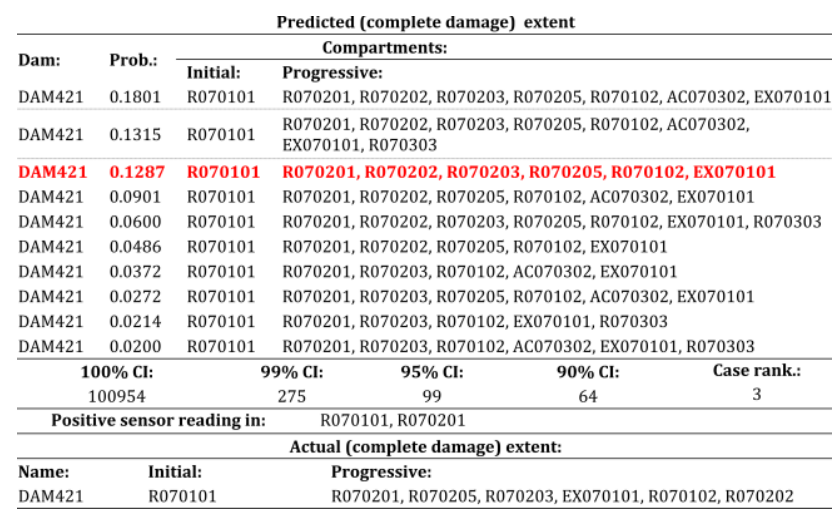

Table 6-9: Inference from flooding sensors, $H_{S}=10 \mathrm{~m}, t=30 \mathrm{~min}$.

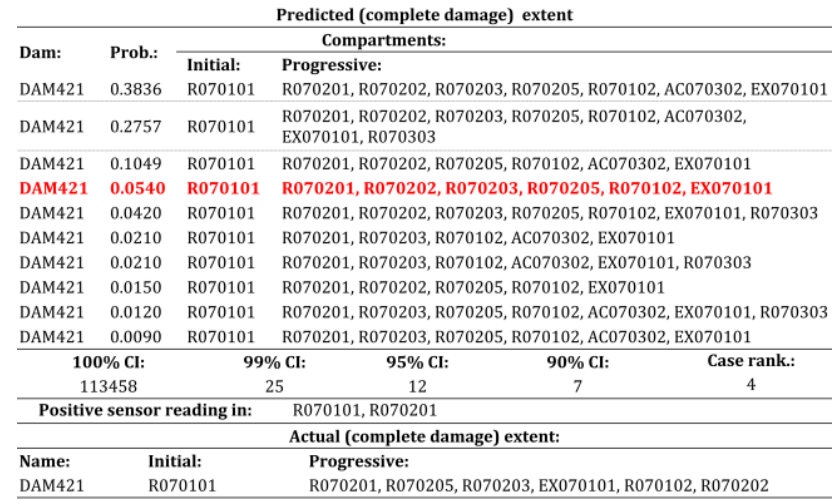

It is noteworthy, however, that the heat maps for the two time-steps, $\mathrm{t}=15 \mathrm{~min}$ and $\mathrm{t}=30 \mathrm{~min}$, are almost identical regardless of the difference in prediction accuracy in terms of unique damage extent (initial- and progressive). This is seen in Figure 6-4 and Figure 6-5, indicating the same flooding boundary, only seeing a larger probability of upflooding in the earlier time-step represented by the more distinct yellow colour due to less evidence.

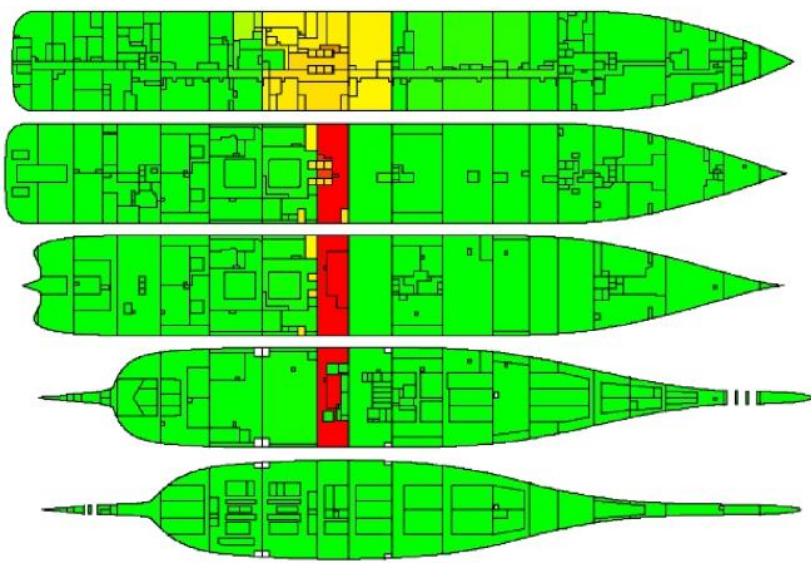

Figure 6-4: Heat-map $-H_{S}=10 \mathrm{~m}, t=15 \mathrm{~min}$.

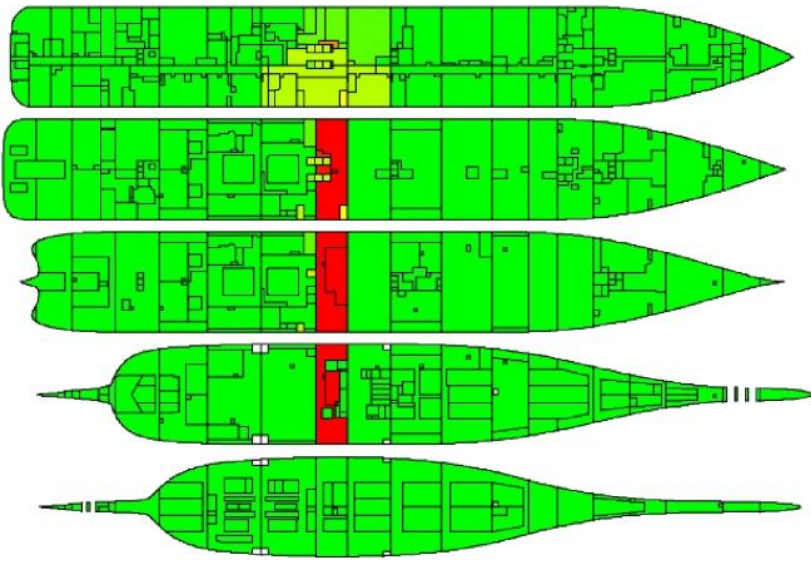

Figure 6-5: Heat-map $-H_{S}=10 \mathrm{~m}, t=30 \mathrm{~min}$.

In comparison to the calm-water case, the wave case sees progressive flooding to additional small Aclass compartments: R070205, R070203 and R070202, all located around midship within the same watertight zone as the initial flooding (Figure 6-6) There is also an increased number of cases corresponding to the $100 \% \mathrm{CI}$, in comparison to the initial extents alone due to the sampled progressive extent realisations from the UGS method, as the 100\% $\mathrm{CI}$ now is representing all possible unique combinations of initial- and progressive extents. 


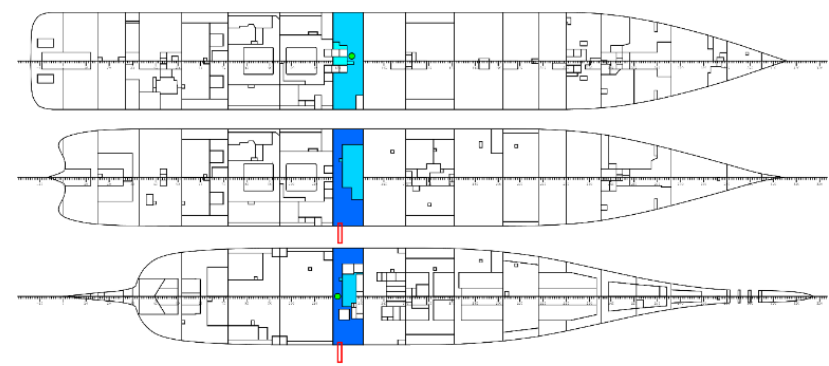

Figure 6-6: Additional progressive flooding extent for wave case.

\subsubsection{Test-case 2 -Calm-water}

Test-case 2, involving striking ship of $250 \mathrm{~m}$ length is illustrated in Figure 6-7. The case extends over 4 watertight zones comprising five initially breached compartments (R100009, R090009, R080116, R100108, R090113) and four progressively flooded compartments (R080201, EX080101, EX100101, R100107).

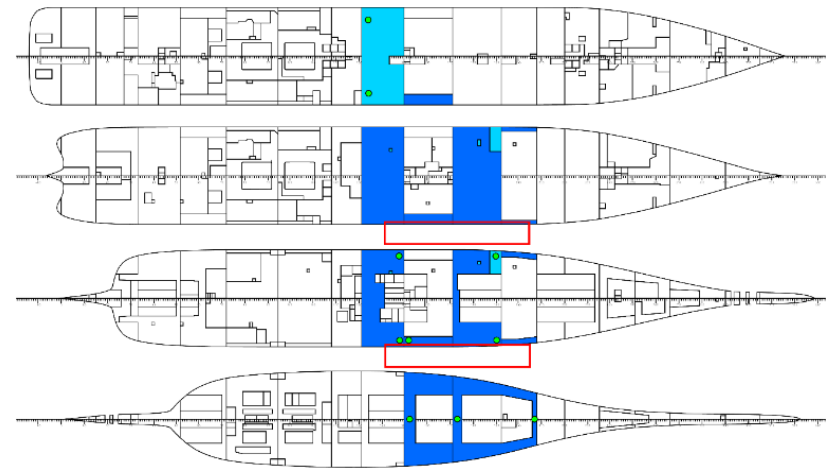

Figure 6-7: Test-case 2, Striking vessel length: $250.0 \mathrm{~m}, H_{S}=0.0 \mathrm{~m}$.

Three sensors appear close to the breach boundary but further examination reveals that the aft- and foremost sensors are located below the breach extent while the mid sensor are located inward of the breach. Cable routing may still be a problem in reality but have not been considered at this point. It should be pointed out that sensors may be fitted with indication of signal loss (which would be the case if the sensor or cabling is damaged). This could be considered evidence in itself and implemented to the likelihood function, indicating that the compartment is part of the initial damage extent. In the following example, we assume that the sensors all are operational and provide proper indication. The initial update incorporating sensor evidence from draught and AIS sensors (Table 6-10) show also here a reduction in the 99\% CI, but less than the reduction seen in the previous test-case corresponding to a considerably smaller striking vessel as was expected. The $99 \%$ CI is now reduced from 10,915 to 3,461 cases, while for the previous case the reduction was from 10,915 to 3,062 .

Table 6-10: Inference from draft and AIS sensor.

\begin{tabular}{|c|c|c|c|c|}
\hline \multicolumn{5}{|c|}{ Predicted initial extent } \\
\hline Damage: & Probability: & & \multicolumn{2}{|l|}{ Compartments: } \\
\hline DAM13 & 0.0366 & & \multicolumn{2}{|c|}{ R180001, R180401 } \\
\hline DAM6 & 0.0266 & & \multicolumn{2}{|l|}{ R180001 } \\
\hline DAM1 & 0.0198 & & \multicolumn{2}{|c|}{ R180001, R180002 } \\
\hline DAM17 & 0.0183 & & \multicolumn{2}{|l|}{ R170101 } \\
\hline DAM65 & 0.0146 & & \multicolumn{2}{|c|}{ R180001, R180002, R180401 } \\
\hline DAM345 & 0.0101 & & \multicolumn{2}{|c|}{ R170101, R170307 } \\
\hline DAM11 & 0.0078 & & \multicolumn{2}{|c|}{ R090009, R090113 } \\
\hline DAM136 & 0.0070 & & \multicolumn{2}{|c|}{ R040112, R040301 } \\
\hline DAM10 & 0.0070 & & \multicolumn{2}{|c|}{ R040112, R040301, R040401 } \\
\hline DAM421 & 0.0070 & & \multicolumn{2}{|l|}{ R070101 } \\
\hline $100 \% \mathrm{Cl}:$ & 99\% Cl: & 95\% CI: & $90 \%$ Cl: & Case rank.: \\
\hline 19225 & 3461 & 1374 & 876 & 4064 \\
\hline \multicolumn{5}{|c|}{ Actual initial extent: } \\
\hline \multirow{2}{*}{\multicolumn{2}{|c|}{ Damage: }} & & \multicolumn{2}{|c|}{ Compartments: } \\
\hline & & & 009, R090009, R0 & 00108, R090113 \\
\hline
\end{tabular}

The calm water case at initial time step $t=$ $5.0 \mathrm{~min}$ (Table 6-11), shows the actual case DAM7043 being part of the ten most likely cases (rank 8 with the probability of 0.005 ). It is further seen that all the ten most likely cases are some realisation of the actual initial case, and the case ranked on top with the probability of 0.82 only varies from the actual case by one compartment (R080201 not included). The excluded compartment has a flooding sensor which is located below the waterplane but does not yet show indication and is the reason for its high rank.

\begin{tabular}{|c|c|c|c|}
\hline \multirow{2}{*}{ Dam: } & \multirow{2}{*}{ Prob.: } & \multicolumn{2}{|c|}{ Compartments: } \\
\hline & & \multirow{2}{*}{$\begin{array}{l}\text { Initial: } \\
\text { R100009, R090009, R080116, } \\
\text { R100108, R090113 }\end{array}$} & Progressive: \\
\hline DAM7043 & 0.8178 & & EX080101, R100107, EX100101 \\
\hline DAM7043 & 0.0912 & $\begin{array}{l}\text { R100009, R090009, R080116, } \\
\text { R100108, R090113 }\end{array}$ & $\begin{array}{l}\text { EX080101, R100107, EX100101, R080202, } \\
\text { R080201 }\end{array}$ \\
\hline DAM7043 & 0.0483 & $\begin{array}{l}\text { R100009, R090009, R080116, } \\
\text { R100108, R090113 }\end{array}$ & $\begin{array}{l}\text { R100201, R100202, EX080101, R100107, } \\
\text { EX100101 }\end{array}$ \\
\hline DAM7043 & 0.0122 & $\begin{array}{l}\text { R100009, R090009, R080116, } \\
\text { R100108, R090113 }\end{array}$ & R100201, EX080101, R100107, EX100101 \\
\hline DAM7043 & 0.0062 & $\begin{array}{l}\text { R100009, R090009, R080116, } \\
\text { R100108, R090113 }\end{array}$ & $\begin{array}{l}\text { R100201, R100202, EX080101, R100107, } \\
\text { EX100101, R080202, R080201 }\end{array}$ \\
\hline DAM7043 & 0.0053 & $\begin{array}{l}\text { R100009, R090009, R080116, } \\
\text { R100108, R090113 }\end{array}$ & EX080101, R100107 \\
\hline DAM7043 & 0.0053 & $\begin{array}{l}\text { R100009, R090009, R080116, } \\
\text { R100108, R090113 }\end{array}$ & R100107, EX100101 \\
\hline DAM7043 & 0.0050 & $\begin{array}{l}\text { R100009, R090009, } \\
\text { R080116, R100108, R090113 }\end{array}$ & EX080101, R100107, EX100101, R080201 \\
\hline DAM7043 & 0.0026 & $\begin{array}{l}\text { R100009, R090009, R080116, } \\
\text { R100108, R090113 }\end{array}$ & $\begin{array}{l}\text { R100201, R100202, EX080101, R100107, } \\
\text { AC100302, EX100101 }\end{array}$ \\
\hline DAM7043 & 0.0015 & $\begin{array}{l}\text { R100009, R090009, R080116, } \\
\text { R100108, R090113 }\end{array}$ & $\begin{array}{l}\text { R100201, EX080101, R100107, EX100101, } \\
\text { R080202, R080201 }\end{array}$ \\
\hline \multicolumn{2}{|c|}{$100 \% \mathrm{CI}:$} & 99\% CI: & Case rank: \\
\hline \multicolumn{2}{|c|}{357757} & 8 & 2 \\
\hline \multicolumn{2}{|c|}{ Positive sensor reading $i$} & \multicolumn{2}{|c|}{$\begin{array}{l}\text { R090009, R100009, R080116, R080116, R090113, R100107, } \\
\text { R100108 }\end{array}$} \\
\hline \multicolumn{2}{|c|}{ Sensor, but no reading: } & R080201 & \\
\hline \multicolumn{4}{|c|}{ Actual (complete damage) extent: } \\
\hline Name: & \multicolumn{2}{|l|}{ Initial: } & Progressive: \\
\hline DAM7043 & \multicolumn{2}{|c|}{$\begin{array}{l}\text { R100009, R090009, R080116, R100108, } \\
\text { R090113 }\end{array}$} & $\begin{array}{l}\text { R080201, EX080101, EX100101, } \\
\text { R100107 }\end{array}$ \\
\hline
\end{tabular}


The progressive flooding rate for the calm-water case is low and the sensor does not submerge within the 30-min simulation time, similar to the initial testcase. The $99 \%$ CI has from the previous posterior update reduced from 3,461 to only 8 cases. The list is maintained virtually the same for the remaining timesteps due to the lack of indication in R080201, only seeing a slight increase of the actual case to rank 3 , and $99 \% \mathrm{CI}$ represented by 5 cases in the final timestep (Table 6-12).

Table 6-12: Inference from flooding sensors, $H_{S}=0 \mathrm{~m}, t=30 \mathrm{~min}$.

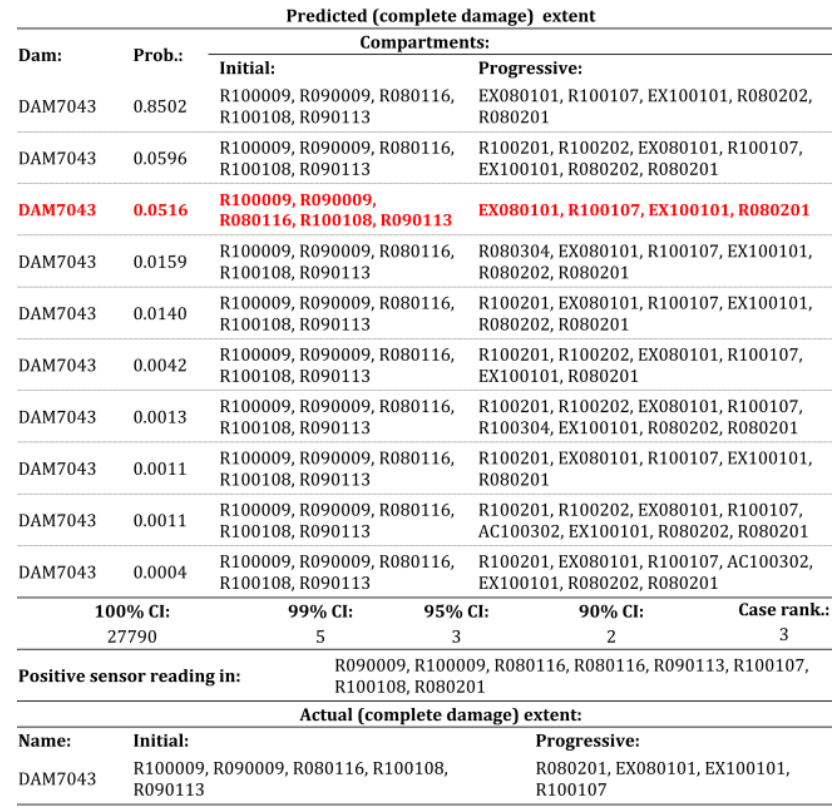

\subsubsection{Test-case 2 - Wave}

For the wave case, time-step $t=5.0 \mathrm{~min}$ (Table 6-13), the actual case is ranked $11^{\text {th }}$ while a slightly different combination of the same initial extent is again ranked on top with a probability of 0.80 . Due to the waves, the progressive flooding rate is increased and the sensor in R080201 indicates flooding at $t=$ $15 \mathrm{~min}$, boosting the actual case to a third rank with a prediction probability of 0.07 . In the following timestep an additional compartment progressively floods, namely R080202, ranking the actual case first with a probability of 0.53 and the $99 \% \mathrm{CI}$ is represented by 45 cases. At the successive time-steps the actual case is top ranked with the probability of 0.55 seen in the final time-step (Table 6-14). The table has been limited to six cases due to large number of sampled compartments in the progressive stage for the remaining cases.
Table 6-13: Inference from flooding sensors, $H_{S}=10 \mathrm{~m}, t=5 \mathrm{~min}$.

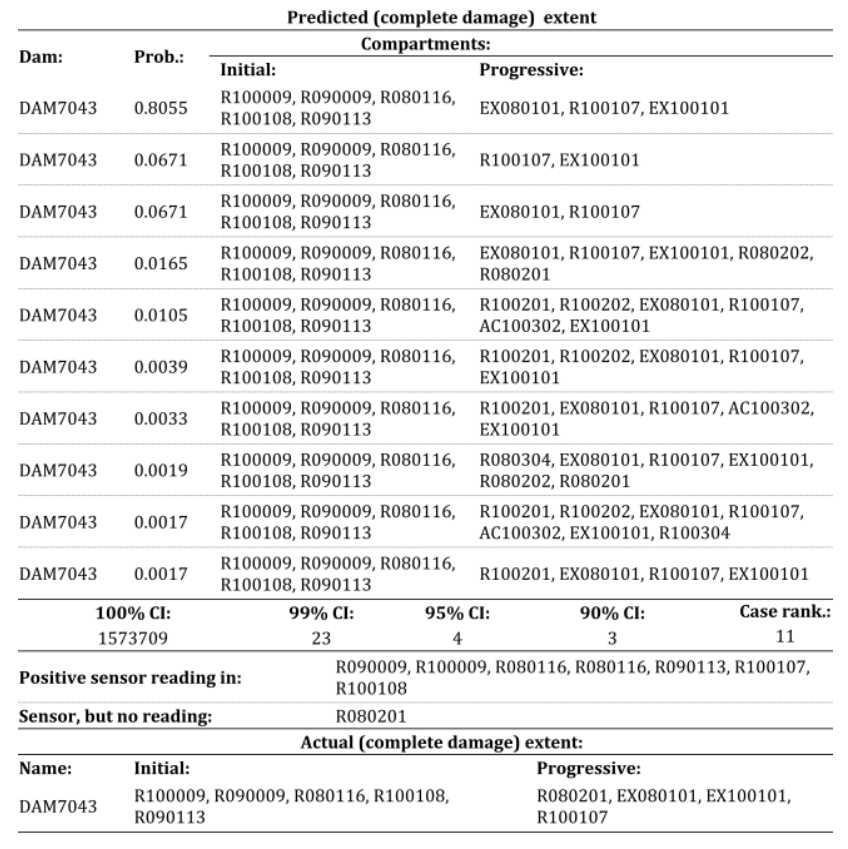

Table 6-14: Test-case 2 - Six most likely cases, $H_{S}=10 \mathrm{~m}, t=30 \mathrm{~min}$

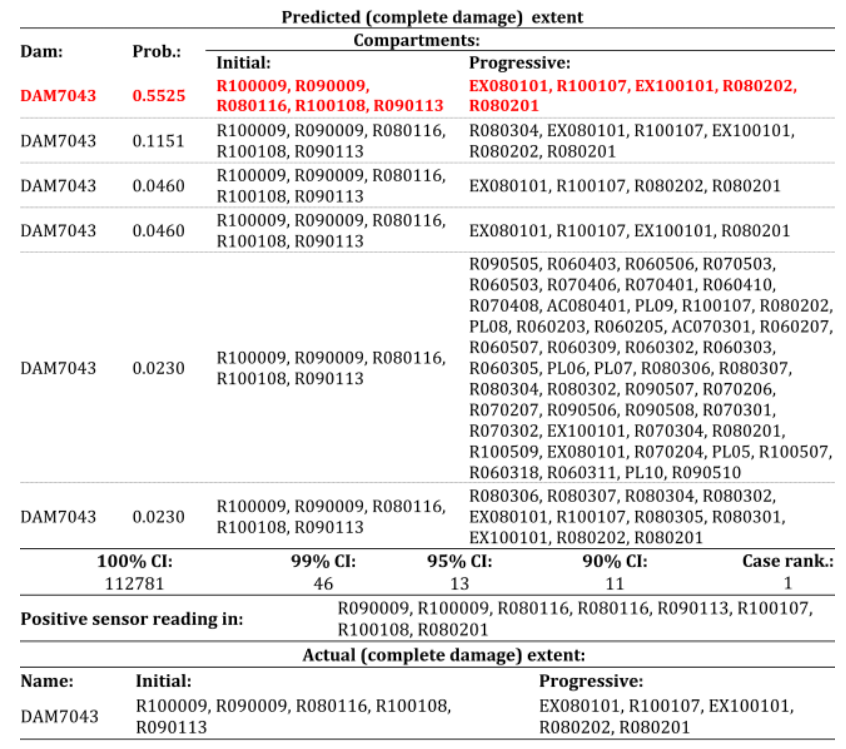

The final extent included with the additional compartment R080202 (minor A-class compartment) is illustrated in Figure 6-8. Heat-maps for both calmwater and the wave case is seen in Figure 6-9 to Figure 6-10 and Figure 6-11 to Figure 6-12 for the initial and last time-step respectively. The figures clearly show that the heat-map presentation indicate the damaged region already from the initial timesteps, providing fast and targeted prediction. The distinct yellow region in Figure 6-11 and Figure 6-12 
is due to the extreme $\mathrm{H}_{\mathrm{S}}$ and as the yellow colour is representing a relatively small probability, i.e. $P \approx$ 0.01 . Different scaling would obviously provide different (colour/heat) for the respective probabilities.

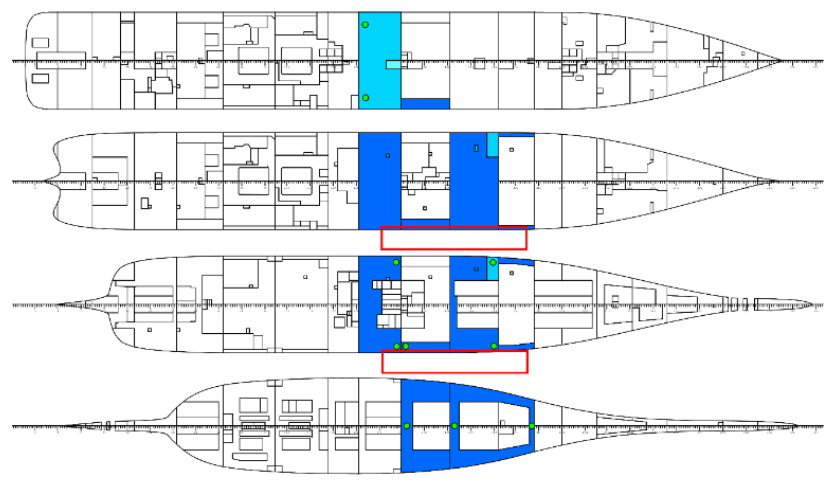

Figure 6-8: Additional progressive extent for Test-case 2, $\mathrm{Hs}=10 \mathrm{~m}$.

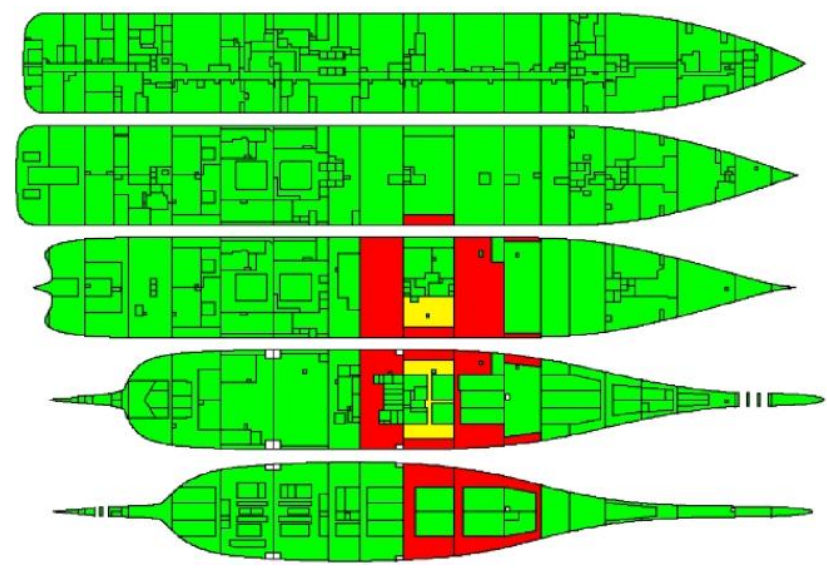

Figure 6-9: Heat-map for Test-case $2-H_{S}=0, t=5$.
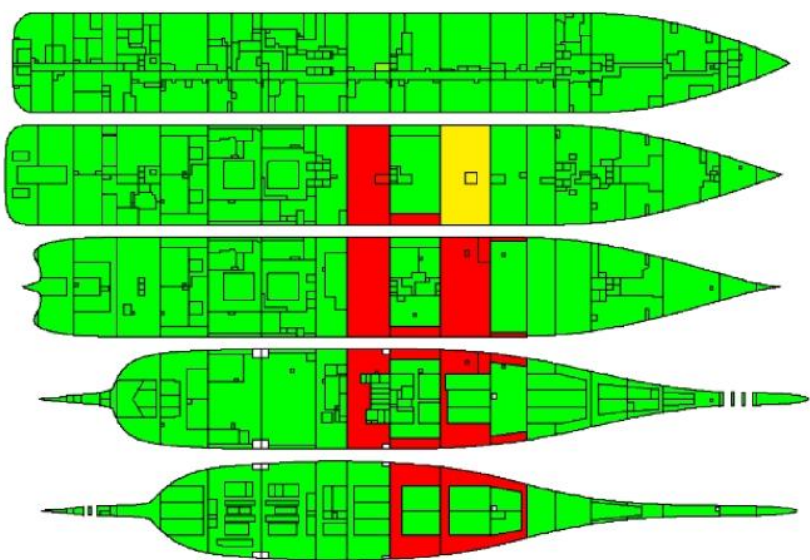

Figure 6-10: Heat-map for Test-case $2-H_{S}=0, t=30$.

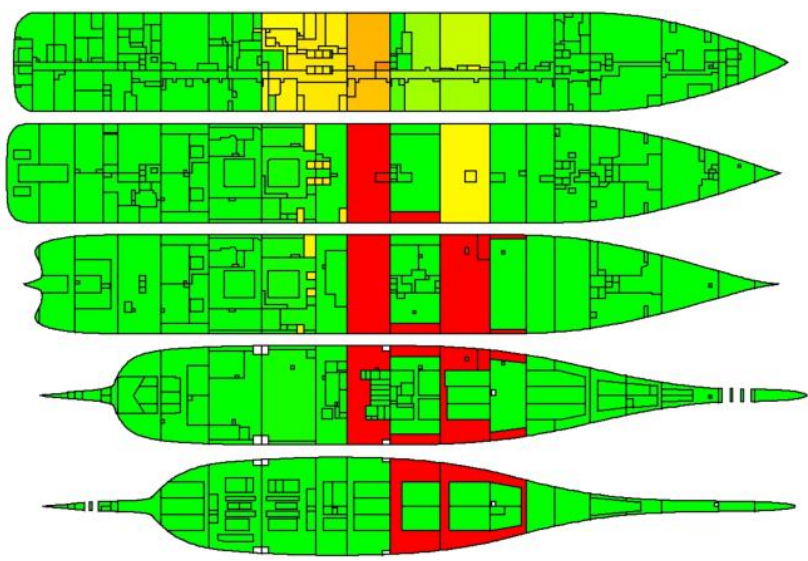

Figure 6-11: Heat-map for Test-case $2-H_{S}=10, t=5$.
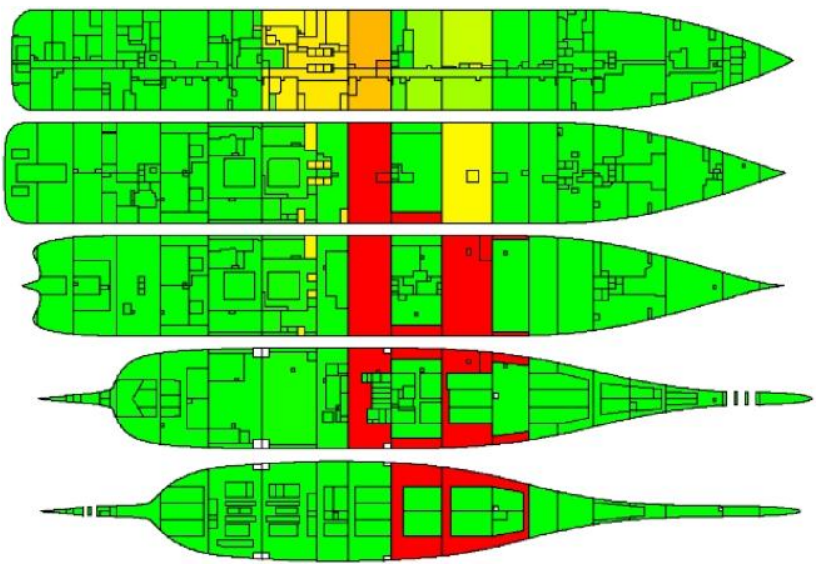

Figure 6-12: Heat-map for Test-case $2-H_{S}=10, t=30$.

\section{SUMMARY AND CONCLUSION}

The foregoing sections detail a fully probabilistic framework developed with the aim to improve realtime information, providing risk-informed situational awareness in flooding emergencies for optimised emergency response. The methodology utilises probabilistic (Bayesian) inference in the form of multi-sensor data fusion techniques for manipulating conditional probability distributions for uncertainty reduction. The method utilizes sequential updates, which is a memory-efficient way of utilising the previous posterior information as the current a-priori belief, which increase the accuracy of the prediction (represented by number of cases within the confidence interval) providing quantitative evidence to support decision-making.

The correct prediction of the flooding extent is essential in survival assessment and in managing 
timely, targeted and efficient emergency response. The heat-maps used to present the results demonstrate that the predictions are converging to the region of the actual damage within the first time-steps in all testcases. Rapid prediction of the compromised area enables fast and targeted deployment of the damage control teams, while the active mitigation measures may be implemented at an early stage with the help of the probabilistic inference of the most likely flooding extent. The predictions allows effective monitoring and management of the openings within the flooding boundary as well as optimised use of other resources such as bilge or ballast systems and cross- and down flooding arrangements. Furthermore, it increases flexibility for use of openings in the areas not affected by the casualty, thus allowing more leeway in managing traffic and, if necessary, facilitating evacuation.

The successful test-cases demonstrate that the methodology accurately identifies the extent and location of the actual flooding casualty even with a sparse flooding sensor array. This, in turn, indicates that the framework may be implemented on a large cruise vessel without any changes to the existing sensor layout (i.e. with as-built sensor arrays), although use of an optimised layout could improve both the accuracy and convergence rate of the prediction. The configuration (i.e. types) and layout of flooding sensors, offering the best trade-offs between accuracy of flooding prediction and complexity, could be a part of the optimisation. The developed method does not rely on a dense array of flooding sensors to produce estimates, because of the use of likelihood functions to allow for combining sensor information from various sources. In a way, the likelihood functions act as filters in a process of screening flooding scenarios identifying common traits and accordingly assigns updated likelihoods. Special attention may be given to the type of flooding sensors, as the framework presented herein does not require pneumatic flooding sensors, which enable inflow-rate estimates. Hence, the flooding sensors may be of a simpler (and less expensive) type, such as limit switches.

On the other hand, the time-variant characteristics, such as time-to-capsize may require the estimate of flooding rates and the flooding sensors providing estimates of inflow-rate. It is important to stress that the present framework, does not provide means for estimating Time-to-Capsize. It does provide, however, the foundations for decision support, which could be extended to accommodate for assessment of the likely outcome of the flooding accident. Needless to say, the accurate prediction of flooding extent, such as presented herein, is a fundamental prerequisite for, and could be of great assistance in, decision making in emergencies, thus saving lives.

An important part of the development relates to the use of time-domain simulations allowing to develop probabilistic models capturing both the probability of initial flooding based on the vertical elevation of the damage breach with respect to the waterplane and conditional on vessel's draught and significant wave height, including the probability of vertical exceedance of the water elevation in the damaged condition of the vessel. The range of probabilistic models has been developed for the specific sample vessel using the state-of-the-art time-domain simulation code PROTEUS3, enabling the simulation of a damaged ship in a dynamic operational climate capturing dynamic variables in greater detail than the traditional static calculation tools.

Representing the waterplane as a mathematical (dynamically changing) plane, establishes a continuous reference to any point within the vessel coordinate system and has been important in the development of a dynamically changing progressive flooding probability. Combined with the UncertainGraph Sampling method, this blend into a robust probabilistic model for progressive flooding stages. An important aspect considered, while modelling progressive flooding, is the status of the internal openings. This entails not only the open/close status but also leaking and collapse under the build-up of floodwater. Hence, the likelihood model developed for the framework takes into account the status of the opening (e.g. open/close) and also its position with respect to the external calm waterplane and the probability of leaking or collapse, both given as distributions around the nominal leak/collapse hydrostatic pressure heads. 
The developed model, by incorporating AIS data, indicates that evidence on striking vessel length provides a global shift in probabilities. This is particularly evident in case of smaller striking vessels. For larger vessels, the data suggest that the full range of damage extent is still possible with both minor as well as major damage extents. It can be argued, however, that additional variables, such as relative speed and heading, would provide further evidence and result in a similar shift in probabilities for larger striking vessel lengths. The challenges relate to the availability of data. The GOALDS database utilised in this development does not contain enough datapoints for such implementation and alternatives are necessary.

It was mentioned in the introduction that the developed framework would be suitable for implementation as a framework for life-cycle flooding risk management. It is evident that such development would entail extensive time-domain simulations and in-depth analysis of all the variables relevant to a flooding scenario. Undoubtedly, such undertaking would require detailed ship-specific knowledge far in excess of that linked to the regulatory framework of SOLAS (2009). Furthermore, it would be well aligned with the riskbased ship design philosophy mentioned in the introduction. In the design stage, the process described in the foregoing could be used to examine in detail the critical flooding scenarios to identify and address local vulnerabilities. Such an approach would allow comprehensive testing of the watertight subdivision, internal openings and flooding sensors layouts. In addition to this, it would offer a platform for systematic verification and validation of the design assumptions.

Furthermore, the sensor data collected during the operation of existing ships could be used as a-priori beliefs. In operation, the probabilistic models could provide a measure of vulnerability to flooding in a way similar to the developments presented in section 1.5.2 (Jasionowski: 2010, 2011). In principle, the vulnerability can be expressed as an average product of probability of damage occurrence (e.g. p-factor) and probability of capsize or sinking (e.g. complement to s-factor). Unlike the static A-index approach the operational vulnerability measure would be a dynamic quantity calculated with the use of evidence from onboard sensors such as door, draught and motion sensors. In summary, the work presented herein lays the foundations for a fully probabilistic methodology for predicting the outcome of flooding casualties and outlines a comprehensive framework for life-cycle flooding risk management.

\section{REFERENCES}

Bitner-Gregersen, E. M., Eide, L. I., Hørte, T., Skjong, R., (2012), “Ship and Offshore Structure Design in Climate Change Perspective", Published with open access at SpringerLink.com, 2013, ISBN 978-3-642-34137-3.

Bulian, G., (2011), "Report detailing derivation of updated probability distributions of collision damage characteristics for passenger ships", Report No. GOALDS-D-3.1-GL- Collision Damage Characteristics rev1 of work package WP3, deliverable D3.1 from the GOAL based Damage Stability (GOALDS) research project, funded by the European Commission, FP7-DG.

DNV GL, (2017), "Environmental conditions and environmental loads", Recommended practice DNVGL-RP-C205, August.

DNV GL, (2018), "Guidance on definition of requirements for sensor system reliability", Internal report No. 2018-0732/0.

Durrant-Whyte H., Henderson T .C. (2016) "Chapter: Multisensor Data Fusion", In: Siciliano B., Khatib O. (eds), Springer Handbook of Robotics, Springer, Cham 2016.

Gagniuc, P.A., (2017), "Markov Chains: From Theory to Implementation and Experimentation”, Book: John Wiley \& Sons, ISBN: 978-1-11938755-8.

Grønlie, Ø. (2004). "Wave Radars - A comparison of different concepts and techniques", Hydro International, volume 8, number 5, June.

International Maritime Organisation (IMO), (2008), "Guidelines for Flooding Detection Systems on Passenger Ships”, MSC.1/Circ.1291 9th of December, Ref. T1/2.04.

International Maritime Organization (IMO), (2009), Reg. II-1/13 of "SOLAS Consolidated Edition 2009", as adopted in IMO Res. MSC 216(82)), 2006.

International Maritime Organization (IMO), (2009), Reg. II-1/13-6 of "SOLAS Consolidated Edition 2009", as adopted in IMO Res. MSC 216(82)), 2006.

International Maritime Organization (IMO), (2009), Reg. II-1/16.2 of "SOLAS Consolidated Edition 2009", as adopted in IMO Res. MSC 216(82)), 2006.

International Maritime Organization, (2009), Reg. I/5 of "SOLAS Consolidated Edition 2009”, as adopted in IMO Res. MSC 216(82)), 2006.

International Maritime Organization, (2009), Reg. II-1/22-1 of "SOLAS Consolidated Edition 2009”, as adopted in IMO Res. MSC 216(82)), 2006.

International Maritime Organization, (2009), Reg. II-1/6 to 8 of "SOLAS Consolidated Edition 2009", as adopted in IMO Res. MSC 216(82)), 2006. 
International Maritime Organization, (2009), Reg. II-1/8-1.3 of "SOLAS Consolidated Edition 2009", as adopted in IMO Res. MSC 216(82)), 2006.

International Maritime Organization, (2009), Reg. V/19 of "SOLAS Consolidated Edition 2009", as adopted in IMO Res. MSC 216(82)), 2006.

International Organization for Standardization (ISO), (1994), “Accuracy (trueness and precision) of measurement methods and results - Part 1: General principles and definitions", ISO standard No. IS0 57251:1994(E).

Jalonen R., Ruponen P., Jasionowski A., Maurier P., Kajosaari M., Papanikolaou A., (2012), "FLOODSTAND - Overview of Achievements", Proceedings of the 11th International Conference on Stability of Ships and Ocean Vehicles, STAB2012, Athens, Greece, 819829.

Jalonen, R., Ruponen, P., Weryk, M., Naar, H., Vaher, S., (2017), “A study on leakage and collapse of non-watertight ship doors under floodwater pressure", Marine Structures Volume 51, January 2017, Pages 188-201.

Jasionowski, A., (2001), "An Integrated Approach to Damage Ship Survivability Assessment", Ph.D. Thesis, Strathclyde University, NAOME, Glasgow, UK.

Jasionowski, A., (2010), "Decision Support for Crisis Management and Emergency Response", Proceedings of the 11th International Ship Stability Workshop, Wageningen Netherlands.

Jasionowski, A., (2011), "Decision support for ship flooding crisis management", Ocean Engineering 38, 1568-1581.

Jasionowski, A., Luhman, H., Bertin, R., Routi, A-L., Cardinale, M., Harper, G., (2015), "Evaluation of risk from watertight doors", report No. 2015-0167 Rev 7 from the EMSA III research project, funded by the European Maritime Safety Agency, EMSA/OP/10/2013.

Karolius B. K., (2019), "Risk-based, sensor-fused detection of flooding casualties for emergency response", Ph.D. Thesis, Maritime Safety Research Centre (MSRC), Strathclyde University, NAOME, Glasgow, UK.

Karolius K. B., Cichowicz, J. and Vassalos, D., (2018), "Modelling of compartment connectivity and probabilistic assessment of progressive flooding stages for a damaged ship", Proceedings of the 17th International Ship Stability Workshop, 10-12 June, Helsinki, Finland.

Lutzen, M., (2001), "Ship Collision Damage", Ph.D. thesis, Technical University of Denmark, Department of Mechanical Engineering.

Lutzen, M., (2002), "Damage Distributions”, Report No. 2-22-D-200101-4 of work package WP2, deliverable D2.2 from the Harmonization of Rules and Design Rational (HARDER) research project, funded by the European Commission, DG XII-BRITE.

Ministry of Infrastructure and Transports (MIT), Marine Casualties Investigative Body, (2012), "Cruise Ship Costa Concordia, Marine Casualty on January 13, 2012, Report on the Safety Technical Investigation", Public investigation report.

Paik, J. K., Sea, J. K., (2007), "A method for progressive structural crashworthiness analysis under collisions and grounding", Thin-Walled Structures 45, 15-23.
Papanikolaou A., Vassalos D., Skjong R., Skovbakke J. J., Jensen J., McGeorge D., (2009), "Risk-Based Ship Design”, Book: Springer, Berlin, ISBN 978-3-540-89041-6.

Papanikolau, P. A., (2007), "Review of Damage Stability of Ships Recent Developments and Trends", Proceedings 10th Int. Symposium on Practical Design of Ships and Other Floating Structures (PRADS) 2007 Houston, October.

Paterson, D., Atzampos, G., (2017), "Analysis of onboard data with regards to probabilities of initial draughts", report No. eSAFE- D1.2.1, Rev. 5.0 of work package WP1, deliverable D1.2.1 from the Enhanced Stability after a flooding event (eSAFE) research project (Joint industry project on Damage Stability for cruise ships).

Pedersen, P. T., Zhang, S., (1999), "Collision Analysis for MS DEXTRA", Paper number (2) SAFER EURORO Spring meeting, NANTES 28 April.

Pedersen, P., (2010), "Review and application of ship collision and grounding analysis procedures”, Marine Structures 23, 241-262.

Penttilä P., Ruponen, P., (2010), "Use of Level Sensors in Breach Estimation for a Damaged Ship", 5th International Conference on Collision and Grounding of Ships, Espoo, Finland.

R Core Team, (2014), "R: A language and environment for statistical computing", R Foundation for Statistical Computing, Vienna, Austria, Online access: http://www.R-project.org/.

Rev. Thomas Bayes, (1763), "Essay Towards Solving a Problem in the Doctrine of Chances", published posthumously in the Philosophical Transactions of the Royal Society.

Ruponen, P., (2007), "Progressive Flooding of a Damaged Passenger Ship", Ph.D. thesis, Helsinki University of Technology, Department of Mechanical Engineering, Ship Laboratory, Espoo.

Ruponen, P., (2017), "On the effects of non-watertight doors on progressive flooding in a damaged passenger ship", Ocean Engineering Volume 130, 15 January 2017, Pages 115-125.

Ruponen, P., Larmela, M., Pennanen, P., (2012), "Flooding Prediction Onboard a Damaged Ship“, Proceedings of the 11th International Conference on the Stability of Ships and Ocean Vehicles 23-28 September, Athens, Greece.

Ruponen, P., Lindroth, D., Pennanen, P., (2015), "Prediction of Survivability for Decision Support in Ship Flooding Emergency", Proceedings of the 12th International Conference on the Stability of Ships and Ocean Vehicles 14-19 June, Glasgow, UK.

Ruponen, P., Pulkkinen, A., Laaksonen, J., (2017), “A Method for Breach Assessment Onboard a Damaged Passenger Ship", Applied Ocean Research 64, 236-248.

Schweder, T., Hjort, N. L., (2016), "Confidence, Likelihood, Probability - Statistical Inference with Confidence Distributions“, Book: Cambridge University press, ISBN 978-0-521-86160-1.

Skjong, R., Bitner-Gregersen, E. M., Cramer, E., Croker, A., Hagen, Ø., Korneliussen, G., Lacasse, S., Lotsberg, I., Nadim, F., Ronold, K. O., (1995), "Guidelines for offshore structural reliability analysis“, DNV report No. 95-2018.

Törnqvist, R., (2003), "Design of Crashworthy Ship Structures", Ph.D. thesis, Technical University of Denmark, Department of Mechanical Engineering. 


\section{APPENDIX I: SCHEMATIC REPRESENTATION OF THE FUSION FRAMEWORK}

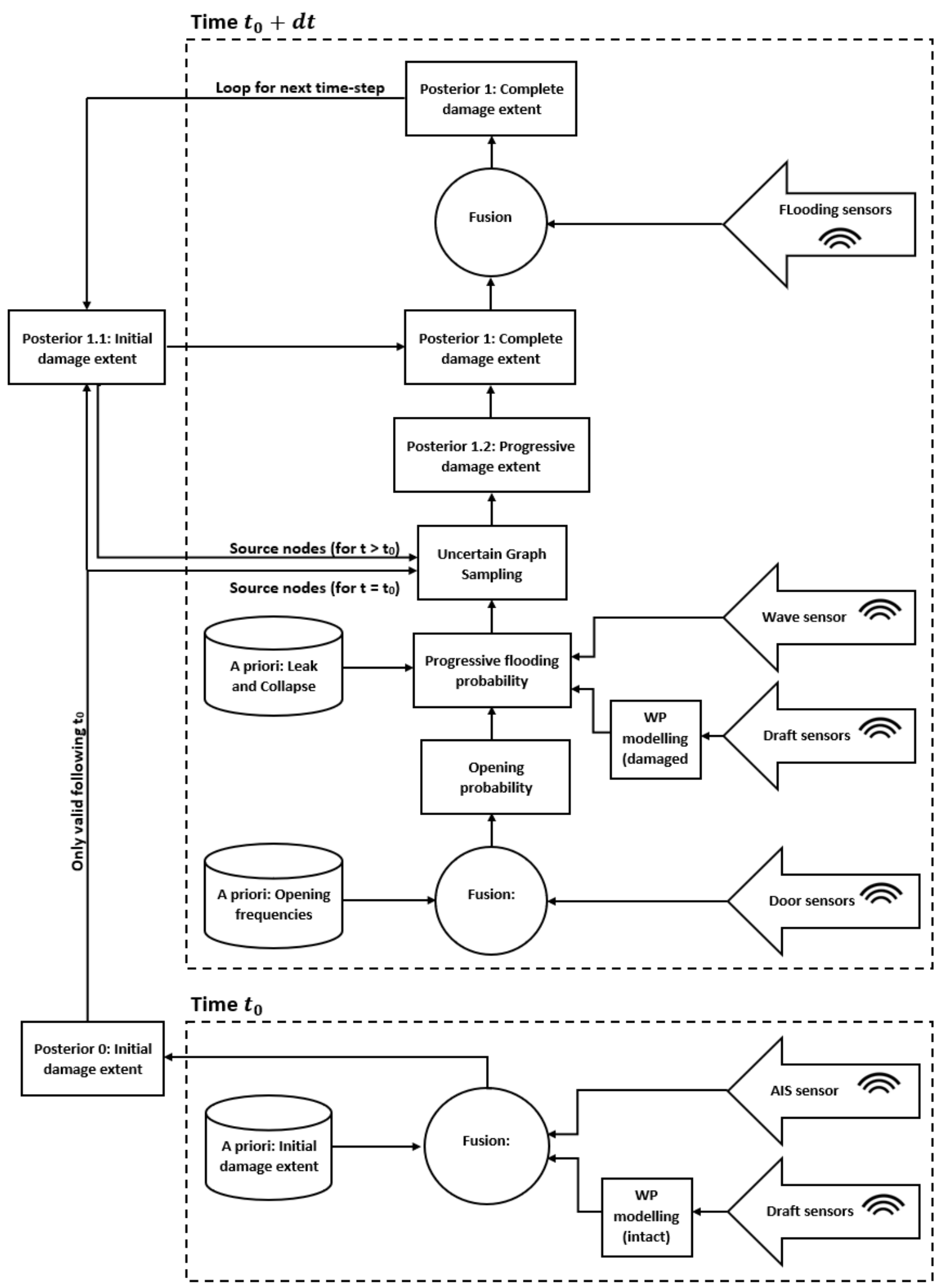

Figure I-1: Schematic layout of the multi-sensor data fusion methodology. 